DESY-06-196

hep-ph/0611101

\title{
Generalized parton distributions for the nucleon in chiral perturbation theory
}

\author{
M. Diehl ${ }^{1}$, A. Manashov ${ }^{2,3}$ and A. Schäfer ${ }^{2}$ \\ ${ }^{1}$ Deutsches Elektronen-Synchroton DESY, 22603 Hamburg, Germany \\ ${ }^{2}$ Institut für Theoretische Physik, Universität Regensburg, 93040 Regensburg, Germany \\ 3 Department of Theoretical Physics, Sankt-Petersburg State University, St.-Petersburg, Russia
}

\begin{abstract}
We complete the analysis of twist-two generalized parton distributions of the nucleon in one-loop order of heavy-baryon chiral perturbation theory. Extending our previous study of the chiral-even isosinglet sector, we give results for chiral-even isotriplet distributions and for the chiral-odd sector. We also calculate the one-loop corrections for the chiral-odd generalized parton distributions of the pion.
\end{abstract}




\section{Contents}

1 Introduction $\quad 2$

2 Chiral-even generalized parton distributions $\quad 2$

3 Heavy-baryon ChPT

4 Chiral even isotriplet operators 6

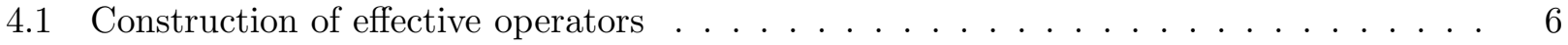

4.2 Power counting for tree and loop graphs . . . . . . . . . . . . . . 8

5 Results for chiral-even isotriplet form factors $\quad 10$

5.1 Relevant operators and graphs . . . . . . . . . . . . . . . . . 10

5.2 Vector form factors . . . . . . . . . . . . . . . . . . . . . 12

5.3 Axial form factors . . . . . . . . . . . . . . . . . . 14

6 Chiral-odd generalized parton distributions $\quad 16$

7 Chiral-odd effective operators $\quad 19$

7.1 Pion-nucleon operators . . . . . . . . . . . . . . . . . . . 19

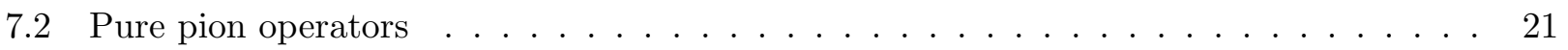

8 Results for chiral-odd form factors $\quad 22$

9 Results for moments of nucleon and pion GPDs 25

10 Summary 31 


\section{Introduction}

Generalized parton distributions (GPDs) provide a unified parameterization of many different aspects of hadron physics [1, 2, 3, 4. Understanding GPDs in detail is therefore tantamount to understanding in large parts the internal structure of hadrons. This motivates extensive experimental programs as well as theoretical work. Details can be found in the reviews [5, 6, 7], which emphasize the different types of physics encoded in these quantities. More recently it has been shown that interesting information about the distribution of transversely polarized quarks in a hadron is contained in GPDs associated with chiral-odd quark operators [8, 9], for which there have been relatively few studies so far.

The extraction of GPDs from experiment is a highly non-trivial task, since in observables the distributions appear only within convolutions. These are relatively simple at leading order in the strong coupling but become increasingly complex at higher orders, see e.g. [10]. In practice one therefore has to use parameterizations of GPDs which are on one hand sufficiently flexible to catch the physics and on the other hand contain only few parameters. In this context, the calculation of moments of GPDs in lattice QCD is expected to become highly important in the future.

The lattice evaluation of these moments, parameterized by the form factors of local matrix elements, is very similar to the case of the usual electromagnetic form factors [11]. The main limitation at present is that lattice calculations with dynamical quarks can only be done for unphysically heavy quarks and thus pions. The mass of the pion affects however the spatial extent of the nucleon and hence its form factors. Therefore, their extrapolation to the physical limit can be fairly non-trivial, and simple linear extrapolations with respect to $m_{\pi}$ or $m_{\pi}^{2}$ could be quite inadequate. Progress in this respect requires an analysis within chiral perturbation theory (ChPT). We have presented such an analysis for the pion GPDs in [12] and for nucleon GPDs in the chiral-even isosinglet sector in [13]. In the present paper we extend this work to the chiral-even isotriplet sector and the chiral-odd sector, giving complete corrections at one-loop accuracy. Calculations of a similar scope have recently been reported in [14, and we will compare our results in detail. There already exists a number of lattice results for moments of GPDs, see [15, 16] and references therein. We do not include any ChPT fits to these in the present paper, but leave them to future lattice studies.

Our paper is organized as follows. In Sections 2, 3 and 6 we collect details about GPD parameterizations, the operator product expansion, and heavy-baryon ChPT that are needed in our analysis. We proceed in each case by constructing the operators within ChPT that match the relevant twist-two operators in QCD, and by identifying the loop corrections which contribute to a given form factor at relative order $O\left(q^{2}\right)$ in the chiral expansion (Sections 4, 5.1] and 7). Results of the corresponding calculations are given for the vector form factors in Section 5.2 , for the axial form factors in Section 5.3, and for the chiral-odd form factors in Section 8. In Section 9 all results are collected and rewritten in terms of the usual parameterization of GPDs. We summarize our main findings in Section 10.

\section{Chiral-even generalized parton distributions}

To begin with let us recall the definitions of generalized parton distributions associated with chiraleven quark operators. For the distributions with definite isospin $I$ in a nucleon one can write

$$
\begin{aligned}
& \int \frac{d \eta}{4 \pi} e^{i x \eta(a P)}\left\langle N_{i}\left(p^{\prime}\right)\left|\bar{q}\left(-\frac{1}{2} \eta a\right) \not d \tau^{A} q\left(\frac{1}{2} \eta a\right)\right| N_{j}(p)\right\rangle \\
& \quad=\tau_{i j}^{A} \frac{1}{2 a P} \bar{u}\left(p^{\prime}\right)\left[\not d H^{I}(x, \xi, t)+\frac{i \sigma^{\mu \nu} a_{\mu} \Delta_{\nu}}{2 M} E^{I}(x, \xi, t)\right] u(p),
\end{aligned}
$$




$$
\begin{aligned}
& \int \frac{d \eta}{4 \pi} e^{i x \eta(a P)}\left\langle N_{i}\left(p^{\prime}\right)\left|\bar{q}\left(-\frac{1}{2} \eta a\right) \not \phi \gamma_{5} \tau^{A} q\left(\frac{1}{2} \eta a\right)\right| N_{j}(p)\right\rangle \\
& =\tau_{i j}^{A} \frac{1}{2 a P} \bar{u}\left(p^{\prime}\right)\left[\not d \gamma_{5} \widetilde{H}^{I}(x, \xi, t)+\frac{a \Delta}{2 M} \gamma_{5} \widetilde{E}^{I}(x, \xi, t)\right] u(p),
\end{aligned}
$$

where $a$ is a light-like auxiliary vector, $M$ is the nucleon mass, and we use the standard kinematical variables $P=\frac{1}{2}\left(p+p^{\prime}\right), \Delta=p^{\prime}-p, t=\Delta^{2}$ and $2 \xi=-(\Delta a) /(P a)$. Wilson lines must be inserted between the quark fields if one is not working in the light-cone gauge $(a A)=0$. We combine the two-dimensional unit matrix $\tau^{0}$ and the triplet of Pauli matrices $\vec{\tau}$ in a four-vector $\tau^{A}=\left(\tau^{0}, \vec{\tau}\right)$, with the matrices acting on the isodoublet of quark fields $q$ or of nucleon states $N$. The isosinglet distributions correspond to $A=0$ and the isotriplet ones to $A=1,2,3$. In terms of individual quark flavors in the proton one has $H^{I=0}=H^{u}+H^{d}$ and $H^{I=1}=H^{u}-H^{d}$, with analogous relations for the other distributions.

The Mellin moments of the GPDs in (1) are related to the matrix elements of the chiral-even local twist-two operators

$$
\begin{aligned}
& \mathcal{O}_{\mu_{1} \mu_{2} \ldots \mu_{n}}^{A}=\underset{\mu_{1} \ldots \mu_{n} \mu_{1} \ldots \mu_{n}}{\mathrm{~T}} \bar{q} \gamma_{\mu_{1}} i \overleftrightarrow{D}_{\mu_{2}} \ldots i \overleftrightarrow{D}_{\mu_{n}} \tau^{A} q, \\
& \widetilde{\mathcal{O}}_{\mu_{1} \mu_{2} \ldots \mu_{n}}^{A}=\underset{\mu_{1} \ldots \mu_{n} \mu_{1} \ldots \mu_{n}}{\mathrm{~T}} \bar{q} \gamma_{\mu_{1}} \gamma_{5} i \stackrel{D^{\prime}}{\mu_{2}} \ldots i \overleftrightarrow{D}_{\mu_{n}} \tau^{A} q
\end{aligned}
$$

with $\overleftrightarrow{D}^{\mu}=\frac{1}{2}\left(\vec{D}^{\mu}-\overleftarrow{D}^{\mu}\right)$. Here $\mathrm{T}$ denotes the subtraction of trace terms in the indicated Lorentz indices and $\mathrm{S}$ denotes symmetrization, normalized as $\mathrm{S}_{\mu_{1} \mu_{2}} t^{\mu_{1} \mu_{2}}=\frac{1}{2}\left(t^{\mu_{1} \mu_{2}}+t^{\mu_{2} \mu_{1}}\right)$. Both operations are conveniently implemented by contraction with the auxiliary vector $a$,

$$
\mathcal{O}_{n}^{A}(a)=a^{\mu_{1}} \ldots a^{\mu_{n}} \mathcal{O}_{\mu_{1} \ldots \mu_{n}}^{A}, \quad \widetilde{\mathcal{O}}_{n}^{A}(a)=a^{\mu_{1}} \ldots a^{\mu_{n}} \widetilde{\mathcal{O}}_{\mu_{1} \ldots \mu_{n}}^{A} .
$$

The local matrix elements can be parameterized as

$$
\begin{aligned}
\left\langle N_{i}\left(p^{\prime}\right)\left|\mathcal{O}_{n}^{A}(a)\right| N_{j}(p)\right\rangle= & \tau_{i j}^{A} \sum_{\substack{k=0 \\
\text { even }}}^{n-1}(a P)^{n-k-1}(a \Delta)^{k} \bar{u}\left(p^{\prime}\right)\left[\not d A_{n, k}^{I}(t)+\frac{i \sigma^{\mu \nu} a_{\mu} \Delta_{\nu}}{2 M} B_{n, k}^{I}(t)\right] u(p) \\
& +\tau_{i j}^{A} \bmod (n+1,2)(a \Delta)^{n} \frac{1}{M} \bar{u}\left(p^{\prime}\right) u(p) C_{n}^{I}(t), \\
\left\langle N_{i}\left(p^{\prime}\right)\left|\widetilde{\mathcal{O}}_{n}^{A}(a)\right| N_{j}(p)\right\rangle= & \tau_{i j}^{A} \sum_{\substack{k=0 \\
\text { even }}}^{n-1}(a P)^{n-k-1}(a \Delta)^{k} \bar{u}\left(p^{\prime}\right)\left[\not d \gamma_{5} \widetilde{A}_{n, k}^{I}(t)+\frac{a \Delta}{2 M} \gamma_{5} \widetilde{B}_{n, k}^{I}(t)\right] u(p),
\end{aligned}
$$

and the moments of the GPDs are given by

$$
\begin{aligned}
\int_{-1}^{1} d x x^{n-1} H(x, \xi, t) & =\sum_{\substack{k=0 \\
\text { even }}}^{n-1}(2 \xi)^{k} A_{n, k}(t)+\bmod (n+1,2)(2 \xi)^{n} C_{n}(t), \\
\int_{-1}^{1} d x x^{n-1} E(x, \xi, t) & =\sum_{\substack{k=0 \\
\text { even }}}^{n-1}(2 \xi)^{k} B_{n, k}(t)-\bmod (n+1,2)(2 \xi)^{n} C_{n}(t), \\
\int_{-1}^{1} d x x^{n-1} \widetilde{H}(x, \xi, t) & =\sum_{\substack{k=0 \\
\text { even }}}^{n-1}(2 \xi)^{k} \widetilde{A}_{n, k}(t), \\
\int_{-1}^{1} d x x^{n-1} \widetilde{E}(x, \xi, t) & =\sum_{\substack{k=0 \\
\text { even }}}^{n-1}(2 \xi)^{k} \widetilde{B}_{n, k}(t),
\end{aligned}
$$


where here and in the following we omit the isospin label $I$ when it is not required. The restriction to even $k$ in (4) and (5) is a consequence of time reversal invariance.

To calculate the chiral corrections to the nucleon form factors in heavy-baryon chiral perturbation theory we work in the Breit frame, where $\boldsymbol{P}=0$. The incoming and outgoing nucleons then have opposite spatial momenta $\boldsymbol{p}^{\prime}=-\boldsymbol{p}=\boldsymbol{\Delta} / 2$ and equal energies, $p_{0}^{\prime}=p_{0}=M \gamma$ with

$$
\gamma=\sqrt{1-\Delta^{2} / 4 M^{2}} .
$$

In terms of the velocity vector $v$, given by $v=(1,0,0,0)$ in the Breit frame, the incoming and outgoing nucleon momenta are given by $p=M \gamma v-\Delta / 2$ and $p^{\prime}=M \gamma v+\Delta / 2$. Note that $(v \Delta)=(v S)=0$. Dirac bilinears can be expressed in terms of the velocity $v_{\mu}$ and the spin operator $S_{\mu}=\frac{1}{2} i \sigma_{\mu \nu} \gamma_{5} v^{\nu}$. Introducing the spinors

$$
u_{v}(p)=\mathcal{N}^{-1} \frac{1+\psi}{2} u(p), \quad u_{v}\left(p^{\prime}\right)=\mathcal{N}^{-1} \frac{1+\psi}{2} u\left(p^{\prime}\right)
$$

with $\mathcal{N}=\sqrt{(1+\gamma) / 2}$, the matrix elements in (4) can be rewritten as [13]

$$
\begin{aligned}
\left\langle N_{i}\left(p^{\prime}\right)\left|\mathcal{O}_{n}^{A}(a)\right| N_{j}(p)\right\rangle= & \tau_{i j}^{A} \sum_{k=-1}^{n-1}(M \gamma)^{n-k-2}(a v)^{n-k-1}(a \Delta)^{k} \\
& \times \bar{u}_{v}\left(p^{\prime}\right)\left[(a \Delta) E_{n, k+1}^{I}(t)+\gamma[(a S),(S \Delta)] M_{n, k}^{I}(t)\right] u_{v}(p), \\
\left\langle N_{i}\left(p^{\prime}\right)\left|\widetilde{\mathcal{O}}_{n}^{A}(a)\right| N_{j}(p)\right\rangle= & \tau_{i j}^{A} \sum_{k=0}^{n-1}(M \gamma)^{n-k-1}(a v)^{n-k-1}(a \Delta)^{k} \\
& \times \bar{u}_{v}\left(p^{\prime}\right)\left[2 \gamma(a S) \widetilde{E}_{n, k}^{I}(t)+\frac{(a \Delta)(S \Delta)}{2 M^{2}} \widetilde{M}_{n, k}^{I}(t)\right] u_{v}(p),
\end{aligned}
$$

where due to time reversal invariance the terms with $E_{n, k+1}$ are only nonzero for odd $k$, whereas those with $M_{n, k}, \widetilde{E}_{n, k}$ and $\widetilde{M}_{n, k}$ are only nonzero for even $k$. The relation between the form factors in (4) and those in (8) is

$$
\begin{aligned}
E_{n, k}(t) & =A_{n, k}(t)+\frac{\Delta^{2}}{4 M^{2}} B_{n, k}(t) \quad \text { for } k<n, \quad E_{n, n}(t)=\gamma^{2} C_{n}(t), \\
M_{n, k}(t) & =A_{n, k}(t)+B_{n, k}(t), \\
\widetilde{E}_{n, k}(t) & =\widetilde{A}_{n, k}(t) \\
\widetilde{M}_{n, k}(t) & =(1+\gamma)^{-1} \widetilde{A}_{n, k}(t)+\widetilde{B}_{n, k},
\end{aligned}
$$

which is readily inverted to

$$
\begin{aligned}
& A_{n, k}(t)=\frac{1}{\gamma^{2}}\left[E_{n, k}(t)-\frac{\Delta^{2}}{4 M^{2}} M_{n, k}(t)\right], \quad B_{n, k}(t)=\frac{1}{\gamma^{2}}\left[M_{n, k}(t)-E_{n, k}(t)\right], \\
& \widetilde{B}_{n, k}(t)=\widetilde{M}_{n, k}(t)-(1+\gamma)^{-1} \widetilde{E}_{n, k}(t) .
\end{aligned}
$$

\section{Heavy-baryon ChPT}

To set our notation, let us briefly review the main ingredients of chiral perturbation theory for heavy baryons, which is an effective theory for the limit $q, m_{\pi} \ll M$, where $q$ is a generic momentum. 
To describe pions we use the nonlinear representation $U(x)=[u(x)]^{2}=\exp \left[i \pi^{a}(x) \tau^{a} / F\right]$, where $F \approx 92 \mathrm{MeV}$ is the pion decay constant in the chiral limit 1 The explicit breaking of chiral symmetry by the quark masses is implemented by the field $\chi(x)$. We assume the isospin limit, where one can replace $\chi(x) \rightarrow m^{2} \tau^{0}$ with the bare pion mass $m$. We will not use external vector or axial vector fields here. The nucleon is described by the heavy-baryon field $N_{v}(x)=\frac{1}{2}(1+\psi) e^{i M_{0}(v x)} N(x)$, where $M_{0}$ is the bare nucleon mass and $v$ the velocity vector. The Fourier transform of $N_{v}(x)$ depends on the residual nucleon momentum, given by the original nucleon momentum minus $M_{0} v$. Important derived quantities are the axial vector field

$$
u_{\mu}=i\left(u^{\dagger} \partial_{\mu} u-u \partial_{\mu} u^{\dagger}\right)=-\frac{1}{F} \partial_{\mu} \pi^{a} \tau^{a}+O\left(\pi^{3}\right)
$$

the connection

$$
\Gamma_{\mu}=\frac{1}{2}\left(u^{\dagger} \partial_{\mu} u+u \partial_{\mu} u^{\dagger}\right)=\frac{i}{4 F^{2}} \epsilon^{a b c} \pi^{a} \partial_{\mu} \pi^{b} \tau^{c}+O\left(\pi^{4}\right)
$$

and

$$
\chi_{ \pm}=u^{\dagger} \chi u^{\dagger} \pm u \chi^{\dagger} u .
$$

Under global chiral transformations, described by unitary matrices $V_{L}$ and $V_{R}$, the different fields transform as

$$
\begin{array}{rlrl}
U & \rightarrow V_{R} U V_{L}^{\dagger}, & \chi & \rightarrow V_{R} \chi V_{L}^{\dagger}, \\
u & \rightarrow V_{R} u H^{\dagger}=H u V_{L}^{\dagger}, & N_{v} \rightarrow H N_{v}, \\
\Gamma_{\mu} & \rightarrow H \Gamma_{\mu} H^{\dagger}+H \partial_{\mu} H^{\dagger}, &
\end{array}
$$

and $u_{\mu}$ and $\chi_{ \pm}$transform homogeneously as

$$
u_{\mu} \rightarrow H u_{\mu} H^{\dagger}, \quad \chi_{ \pm} \rightarrow H \chi_{ \pm} H^{\dagger} .
$$

The unitary matrix $H$ depends on $V_{L}, V_{R}$ and on $U(x)$ and therefore has an $x$ dependence. With the connection $\Gamma_{\mu}$ one can construct the covariant derivative $\nabla_{\mu}$. It acts as $\nabla_{\mu} X=\partial_{\mu} X+\Gamma_{\mu} X$ on quantities like $N_{v}$, which transform with a factor $H$ on their left, and as $\left[\nabla_{\mu}, Y\right]=\partial_{\mu} Y+\left[\Gamma_{\mu}, Y\right]$ on quantities like $u_{\mu}$, which transform with $H$ on the left and with $H^{\dagger}$ on the right. Corresponding derivatives acting to the left are $Z \overleftarrow{\nabla}=Z \overleftarrow{\partial}-Z \Gamma_{\mu}$ and $[Y, \overleftarrow{\nabla}]=Y \overleftarrow{\partial}-\left[Y, \Gamma_{\mu}\right]$, where $Z$ transforms with a factor $H^{\dagger}$ on its right.

The effective Lagrangian for the theory contains a pure pion piece and a piece describing the nucleon and its interaction with pions, $\mathcal{L}_{\text {eff }}=\mathcal{L}_{\pi}+\mathcal{L}_{\pi N}$. Expanding in powers of $q$ one has

$$
\mathcal{L}_{\pi}=\mathcal{L}_{\pi}^{(2)}+\mathcal{L}_{\pi}^{(4)}+\ldots, \quad \mathcal{L}_{\pi N}=\mathcal{L}_{\pi N}^{(1)}+\mathcal{L}_{\pi N}^{(2)}+\ldots
$$

with [17, 18]

$$
\begin{aligned}
& \mathcal{L}_{\pi}^{(2)}=\frac{F^{2}}{4} \operatorname{Tr}\left(u_{\mu} u^{\mu}+\chi_{+}\right), \\
& \mathcal{L}_{\pi}^{(4)}=\frac{l_{3}}{16}\left(\operatorname{Tr} \chi_{+}\right)^{2}+\frac{l_{4}}{16}\left\{2 \operatorname{Tr} \chi_{+} \operatorname{Tr}\left(u_{\mu} u^{\mu}\right)+2 \operatorname{Tr}\left(\chi_{-}^{2}\right)-\left(\operatorname{Tr} \chi_{-}\right)^{2}\right\}+\ldots,
\end{aligned}
$$

\footnotetext{
${ }^{1}$ Our convention is that uppercase indices of $\tau$ as in (11) run from 0 to 3, whereas lowercase ones run from 1 to 3 .
} 
and [19]

$$
\begin{aligned}
\mathcal{L}_{\pi N}^{(1)}= & \bar{N}_{v} \\
\mathcal{L}_{\pi N}^{(2)}= & \bar{N}_{v} \\
& \left\{\frac{(v \nabla)^{2}-\nabla^{2}}{2 M_{0}}-\frac{i g_{0}}{2 M_{0}}\{(\nabla S),(v u)\}+c_{1} \operatorname{Tr} \chi_{+}\right. \\
& \left.+\left(c_{2}-\frac{g_{0}^{2}}{8 M_{0}}\right)(v u)^{2}+c_{3} u_{\mu} u^{\mu}+\left(c_{4}+\frac{1}{4 M_{0}}\right)\left[S^{\mu}, S^{\nu}\right] u_{\mu} u_{\nu}\right\} N_{v},
\end{aligned}
$$

where $g_{0}$ is the nucleon axial-vector coupling in the chiral limit and the $l_{i}$ and $c_{i}$ are further low-energy

constants. The terms not displayed in $\mathcal{L}_{\pi}^{(4)}$ couple to at least four pion fields and will not be needed in our calculations.

For calculating nucleon matrix elements in the Breit frame we need the residual momenta of the incoming and outgoing nucleon,

$$
r=p-M_{0} v=w v-\Delta / 2, \quad r^{\prime}=p^{\prime}-M_{0} v=w v+\Delta / 2
$$

with

$$
w=M(\gamma-1)+\delta M=-\frac{\Delta^{2}}{8 M}-4 c_{1} m^{2}+O\left(q^{3}\right),
$$

where $\delta M=M-M_{0}$ is the nucleon mass shift. Using the spinors (7) one obtains a matrix element as $[20]$

$$
\left\langle p^{\prime}|\mathcal{O}| p\right\rangle=\mathcal{N}^{2} Z_{N} \bar{u}_{v}\left(p^{\prime}\right) G_{\mathcal{O}}\left(r^{\prime}, r\right) u_{v}(p),
$$

where $G_{\mathcal{O}}\left(r^{\prime}, r\right)$ is the truncated Green function for external heavy-baryon fields $\bar{N}_{v}, N_{v}$ and the operator $\mathcal{O}$ in the effective theory. $Z_{N}$ is the heavy-baryon field renormalization constant,

$$
Z_{N}=1-\frac{3 m^{2} g_{0}^{2}}{2(4 \pi F)^{2}}-\frac{9 m^{2} g_{0}^{2}}{4(4 \pi F)^{2}} \log \frac{m^{2}}{\mu^{2}}-8 m^{2} d_{28}^{r}(\mu)+O\left(q^{3}\right)
$$

where $d_{28}^{r}(\mu)$ is a low-energy constant in the Lagrangian $\mathcal{L}_{\pi N}^{(3)}$ given in [21].

\section{Chiral even isotriplet operators}

\subsection{Construction of effective operators}

To find the operators in the effective theory which match the quark-gluon operators (2) in QCD we generalize the construction of [13] to the isotriplet sector. The relevant effective operators contain a part $\mathcal{O}_{\pi}$ which involves only pion fields (and couples to the nucleon via interactions from $\mathcal{L}_{\pi N}$ ) and a part $\mathcal{O}_{\pi N}$ that is bilinear in the nucleon field. We thus have

$$
\mathcal{O}_{n}^{A}(a) \cong \mathcal{O}_{n, \pi}^{A}(a)+\mathcal{O}_{n, \pi N}^{A}(a), \quad \widetilde{\mathcal{O}}_{n}^{A}(a) \cong \widetilde{\mathcal{O}}_{n, \pi}^{A}(a)+\widetilde{\mathcal{O}}_{n, \pi N}^{A}(a),
$$

where for the pure pion operators $\mathcal{O}_{n, \pi}^{A}(a)$ and $\widetilde{\mathcal{O}}_{n, \pi}^{A}(a)$ we will use the form given in [12]. The pion-nucleon operators $\mathcal{O}_{n, \pi N}^{A}(a)$ and $\widetilde{\mathcal{O}}_{n, \pi N}^{A}(a)$ are conveniently constructed by first matching the operators

$$
\left(\mathcal{O}_{n}^{R}(a)\right)_{i j}=\bar{q}_{j} \not \frac{1+\gamma_{5}}{2}(i a \overleftrightarrow{D})^{n-1} q_{i}, \quad\left(\mathcal{O}_{n}^{L}(a)\right)_{i j}=\bar{q}_{j} \not \frac{1-\gamma_{5}}{2}(i a \overleftrightarrow{D})^{n-1} q_{i}
$$


where $i$ and $j$ are isospin indices. They involve quarks of definite chirality and transform as

$$
\mathcal{O}_{n}^{R}(a) \rightarrow V_{R} \mathcal{O}_{n}^{R}(a) V_{R}^{\dagger} \quad \mathcal{O}_{n}^{L}(a) \rightarrow V_{L} \mathcal{O}_{n}^{L}(a) V_{L}^{\dagger}
$$

unter chiral rotations. Parity transforms $\mathcal{O}_{n}^{R}(a)$ and $\mathcal{O}_{n}^{L}(a)$ into each other. The corresponding effective operators that are bilinear in the nucleon field can be written in the form

$$
\left(Q_{n}^{R}(a)\right)_{i j}=\left(\bar{N}_{v} \mathcal{O}_{1} u^{\dagger}\right)_{j}\left(u \mathcal{O}_{2} N_{v}\right)_{i}, \quad\left(Q_{n}^{L}(a)\right)_{i j}=\left(\bar{N}_{v} \mathcal{O}_{1}^{\prime} u\right)_{j}\left(u^{\dagger} \mathcal{O}_{2}^{\prime} N_{v}\right)_{i}
$$

where $\mathcal{O}_{1}, \mathcal{O}_{2}$ transform like $u_{\mu}$ under chiral rotations and $\mathcal{O}_{1}^{\prime}, \mathcal{O}_{2}^{\prime}$ are related to them by parity. The vector and axial vector operators are then readily obtained as

$$
\mathcal{O}_{n, \pi N}^{A}(a)=\operatorname{Tr} \tau^{A}\left\{Q_{n}^{R}(a)+Q_{n}^{L}(a)\right\} \quad \widetilde{\mathcal{O}}_{n, \pi N}^{A}(a)=\operatorname{Tr} \tau^{A}\left\{Q_{n}^{R}(a)-Q_{n}^{L}(a)\right\}
$$

and will involve the combinations

$$
\tau_{e \pm}^{A}=u^{\dagger} \tau^{A} u \pm u \tau^{A} u^{\dagger}
$$

where the subscript $e$ indicates that they occur in chiral even operators. In the isosinglet case one has simply $\tau_{e+}^{0}=2 \tau^{0}$ and $\tau_{e-}^{0}=0$, whereas the isotriplet combinations

$$
\tau_{e+}^{a}=2 \tau^{a}+\frac{1}{F^{2}} \pi^{b}\left(\pi^{a} \tau^{b}-\pi^{b} \tau^{a}\right)+O\left(\pi^{4}\right) \quad \tau_{e-}^{a}=-\frac{2}{F} \epsilon^{a b c} \pi^{b} \tau^{c}+O\left(\pi^{3}\right)
$$

involve an even or odd number of pion fields, respectively. The operators $\mathcal{O}_{1}, \mathcal{O}_{2}$ can be constructed from the fields $u_{\mu}$ and $\chi_{ \pm}$, and from the covariant derivatives introduced in Section 3. One can rearrange the covariant derivatives in $Q_{n}^{R}(a)$ and $Q_{n}^{L}(a)$ to act either as total derivatives $\partial_{\mu}$ on the product of all fields or in the antisymmetric form $\overleftrightarrow{\nabla}_{\mu}=\frac{1}{2}\left(\vec{\nabla}_{\mu}-\overleftarrow{\nabla}_{\mu}\right)$, where $\vec{\nabla}_{\mu}=\vec{\partial}_{\mu}+\Gamma_{\mu}$ acts on the product of all fields to the right and $\overleftarrow{\nabla}_{\mu}=\overleftarrow{\partial}_{\mu}-\Gamma_{\mu}$ on the product of all fields to the left. The operators $Q_{n}^{R}(a)$ and $Q_{n}^{L}(a)$ are tensors having $n$ indices contracted with the auxiliary vector $a$. Other than $\partial_{\mu}, \overleftrightarrow{\nabla}_{\mu}$ and $u_{\mu}$ these tensors can contain the vectors $v_{\mu}$ and $S_{\mu}$ and the totally antisymmetric tensor. The number of spin vectors can be changed using the identities

$$
\left\{S_{\lambda}, S_{\mu}\right\}=\frac{1}{2}\left(v_{\lambda} v_{\mu}-g_{\lambda \mu}\right), \quad\left[S_{\lambda}, S_{\mu}\right]=i \epsilon_{\lambda \mu \nu \rho} v^{\nu} S^{\rho} \quad S_{\lambda}=-\frac{i}{2} \epsilon_{\lambda \mu \nu \rho} v^{\mu}\left[S^{\nu}, S^{\rho}\right]
$$

where our convention for the totally antisymmetric tensor is $\epsilon_{0123}=1$. For the operators under discussion we chose a basis where $S_{\mu}$ appears at most linearly, or quadratically as the commutator $\left[S_{\lambda}, S_{\mu}\right]$. For counting powers of $q$ one associates chiral dimension 1 to $\partial_{\mu}, \overleftrightarrow{\nabla}_{\mu}, u_{\mu}$ and chiral dimension 2 to $\chi_{ \pm}$.

We now make all factors of $(a v)$ explicit and write

$$
\mathcal{O}_{n, \pi N}^{A}(a)=\sum_{k=0}^{n} M^{n-k-1}(a v)^{n-k} \mathcal{O}_{n, k}^{A}(a),
$$

where $\mathcal{O}_{n, k}^{A}(a)$ is free of factors $(a v)$. For contracting the $k$ vectors $a^{\mu}$ in $\mathcal{O}_{n, k}^{A}(a)$ one can use $S_{\mu}$ only once, so that this operator contains at least $k-1$ vectors $\partial_{\mu}, \overleftrightarrow{\nabla}_{\mu}$ or $u_{\mu}$. Thus we can further decompose

$$
\mathcal{O}_{n, k}^{A}(a)=\sum_{i=-1}^{\infty} M^{-i} \mathcal{O}_{n, k, i}^{A}(a),
$$

where $\mathcal{O}_{n, k, i}^{A}(a)$ has chiral dimension $k+i$. For $\widetilde{\mathcal{O}}_{n, \pi N}^{A}(a)$ one has a decomposition in full analogy to (31) and (32). 
Table 1: Overview of contributions to the chiral even form factors. The restriction in the second column is due to time reversal invariance. $N_{\Delta}$ is the number of factors $(a \Delta)$ and $(S \Delta)$ in the decomposition (8). The indices of the operators must satisfy $l \geq k$ and $i \geq 0$, and the corresponding graphs contribute to the form factor at order $O\left(q^{d}\right)$ with $d \geq D_{l+1, i-1}-N_{\Delta}$ and $D_{l+1, i-1}$ from (33).

\begin{tabular}{cccc}
\hline \hline form factor & $k$ & $N_{\Delta}$ & operators \\
\hline$E_{n, k+1}$ & odd & $k+1$ & $\mathcal{O}_{n, l+1, i-1}$ \\
$M_{n, k}$ & even & $k+1$ & $\mathcal{O}_{n, l+1, i-1}$ \\
$\widetilde{E}_{n, k}$ & even & $k$ & $\widetilde{\mathcal{O}}_{n, l+1, i-1}$ \\
$\widetilde{M}_{n, k}$ & even & $k+2$ & $\widetilde{\mathcal{O}}_{n, l+1, i-1}$ \\
\hline \hline
\end{tabular}

\subsection{Power counting for tree and loop graphs}

As shown in [13] the chiral dimension of a graph with two external nucleon legs and insertion of the operator $\mathcal{O}_{n, k, i}^{A}(a)$ or $\widetilde{\mathcal{O}}_{n, k, i}^{A}(a)$ is

$$
D_{k, i}=2 L+k+i+\sum_{j=1}^{N_{\pi}}\left(\operatorname{dim} V_{\pi}(j)-2\right)+\sum_{j=1}^{N_{\pi N}}\left(\operatorname{dim} V_{\pi N}(j)-1\right),
$$

where $L$ is the number of loops (with $L=0$ for tree graphs). $V_{\pi}(j)$ and $V_{\pi N}(j)$ respectively denote the $j$ th vertex from $\mathcal{L}_{\pi}$ and $\mathcal{L}_{\pi N}$ in the graph, $N_{\pi}$ and $N_{\pi N}$ are the corresponding total numbers of vertices, and $I_{\pi}$ and $I_{N}$ are the numbers of pion and nucleon propagators. Corrections to the nucleon propagator from higher orders of $\mathcal{L}_{\pi N}$ are counted as a nucleon-nucleon vertex and are accompanied by two (leading-order) nucleon propagators on either side. Notice that $r^{\mu}+r^{\prime \mu}=2 w v^{\mu}$ is of order $O\left(q^{2}\right)$ and thus one order higher than the generic power associated with a residual nucleon momentum. A graph with chiral dimension $D_{k, i}$ can thus generate contributions to a nucleon matrix element of order $O\left(q^{d}\right)$ with $d \geq D_{k, i}$. Since $\mathcal{O}_{n, k, i}^{A}(a)$ is accompanied by a factor $(a v)^{n-k}$ in (31) it can only contribute to form factors with at least $n-k$ powers of $(a v)$ in the decomposition (8) of the nucleon matrix element. Taking into account the number $N_{\Delta}$ of factors $(a \Delta)$ and $(S \Delta)$ in that decomposition, one can establish the order in the chiral expansion to which a given operator can contribute to a form factor. The result is given in Table 1.

Throughout this paper we refer to orders $O\left(q^{d}\right)$ in the chiral expansion of a given form factor rather than the expansion of the corresponding matrix element. This is most convenient for the problem at hand, since the chiral order of matrix elements increases with the order $n$ of the operator, whereas the chiral order of the form factors has as a natural point of reference the order $O\left(q^{0}\right)$ from tree-level insertions of operators with the lowest chiral dimension at given $n$.

The contributions of the operators $\mathcal{O}_{n, k, i}^{A}(a)$ and $\widetilde{\mathcal{O}}_{n, k, i}^{A}(a)$ at tree level are readily evaluated. The tree level graphs do not contain pions, so that one can replace

$$
\begin{aligned}
& u^{\mu} \rightarrow 0, \quad \partial^{\mu} \rightarrow i \Delta^{\mu}, \quad \overleftrightarrow{\nabla}^{\mu} \rightarrow-i w v^{\mu}, \\
& \tau_{e+}^{A} \rightarrow 2 \tau^{A}, \quad \tau_{e-}^{A} \rightarrow 0, \quad \chi_{+} \rightarrow 2 m^{2} \tau^{0}, \quad \chi_{-} \rightarrow 0 .
\end{aligned}
$$

Operators with $\overleftrightarrow{\nabla}_{\mu}$ do not contribute to the form factors at leading order since $w$ is of order $O\left(q^{2}\right)$. The different types of higher-order contributions to the form factors are discussed in Section 3.2 of [13. In the results we give for the form factors, we lump them all into coefficients describing the $m^{2}$ 


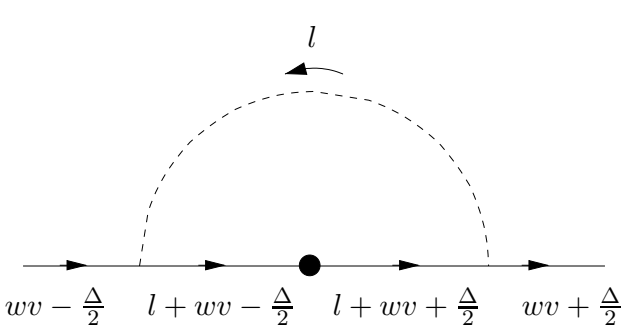

a

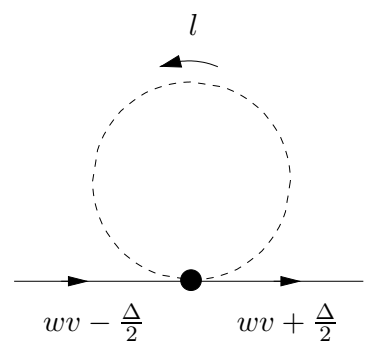

$\mathrm{b}$

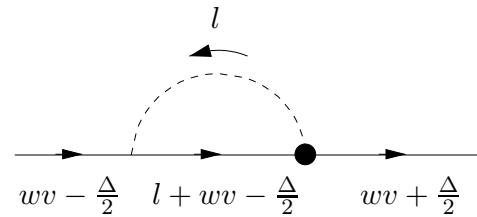

C

Figure 1: One-loop graphs with the insertion of a pion-nucleon operator $\mathcal{O}_{n, \pi N}(a)$ or $\widetilde{\mathcal{O}}_{n, \pi N}(a)$, which is denoted by a black blob. Not shown is the analog of graph c with residual momentum $l+w v+\Delta / 2$ of the intermediate nucleon line.

and $t$ corrections from tree graphs, except for the terms proportional to $g_{0}^{2}$ in the expression (22) of the wave function renormalization constant $Z_{N}$, which we combine with the terms due to loop graphs.

The one-loop graphs with pion-nucleon operator insertions are shown in Fig. 1. The construction of operators detailed in Section 4.1 allows one to easily track the origin of factors $\Delta_{\mu}$ which arise from a graph and must match the factors in the form factor decomposition (8). For this we use that the denominators of the pion and nucleon propagators are $\left(l^{2}-m^{2}+i 0\right)$ and $(l v+w+i 0)$, respectively, so that the loop integration turns tensors $l_{\mu_{1}} \ldots l_{\mu_{j}}$ into tensors constructed from $v_{\mu}$ and $g_{\lambda \mu}$. We find that with the leading-order (LO) interactions from $\mathcal{L}_{\pi N}^{(1)}$ and the next-to-leading (NLO) interactions from $\mathcal{L}_{\pi N}^{(2)}$ a factor $\Delta_{\mu}$ which is not contracted to $\Delta^{2}$ (and hence can be contracted with $a^{\mu}$ or $S^{\mu}$ ) can only originate from [13]

1. a total derivative $\partial_{\mu}$ in the operator insertion,

2. a term $(l v)(S \Delta)$ due to an NLO pion-nucleon vertex,

3. or a term $(l \Delta)$ due to an NLO nucleon propagator correction.

We further find that two factors of $\Delta_{\mu}$ which are not contracted to $\Delta^{2}$ can originate as $(l \Delta)(\Delta S)$ from the NNLO pion-nucleon vertex generated by the term

$$
-\frac{g_{0}}{4 M_{0}^{2}} \bar{N}_{v}\{(\overleftarrow{\nabla} S)(u \vec{\nabla})+(\overleftarrow{\nabla} u)(S \vec{\nabla})\} N_{v}
$$

of the Lagrangian $\mathcal{L}_{\pi N}^{(3)}$ given in [21].

In the next section we will see that $\mathcal{O}_{n, l+1, i-1}^{A}(a)$ and $\widetilde{\mathcal{O}}_{n, l+1, i-1}^{A}(a)$ with $i=0,1,2$ have at most $l+i$ total derivatives $\partial_{\mu}$. A one-loop graph with insertion of such an operator and pion-nucleon interactions up to NNLO must therefore satisfy

$$
l+i+\sum_{j=1}^{N_{\pi N}}\left(\operatorname{dim} V_{\pi N}(j)-1\right) \geq N_{\Delta}
$$

in order to produce the number $N_{\Delta}$ of factors $\Delta_{\mu}$ required to contribute to the form factors in Table 1. For $i>2$ and for pion-nucleon interactions higher than NNLO this inequality is trivially fulfilled. With the power counting established in the table, one then finds that the one-loop contributions from pion-nucleon operators for all form factors start at order $O\left(q^{2}\right)$. 


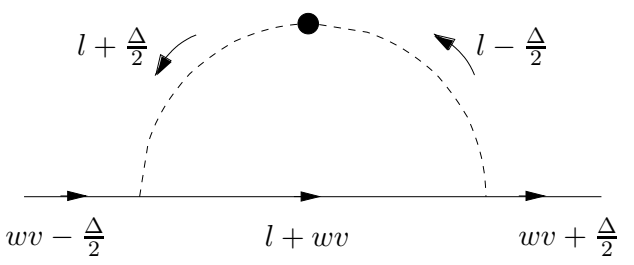

a

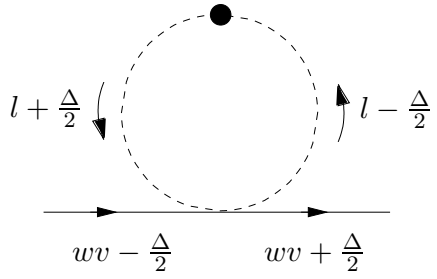

$\mathrm{b}$

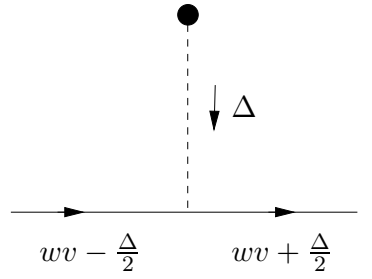

$\mathrm{C}$

Figure 2: $\mathbf{a}$ and $\mathbf{b}$ : One-loop graphs with the insertion of the pion operator $\mathcal{O}_{n, \pi}(a)$. c: Tree graph with the insertion of the pion operator $\widetilde{\mathcal{O}}_{n, \pi}(a)$. The operator insertions are denoted by black blobs.

Let us finally return to the contributions to the nucleon matrix elements from the pure pion operators $\mathcal{O}_{n, \pi}^{A}(a)$ and $\widetilde{\mathcal{O}}_{n, \pi}^{A}(a)$. Their chiral dimension is [13]

$$
D_{\pi}=2 L-1+d_{\pi}+\sum_{j=1}^{N_{\pi}}\left(\operatorname{dim} V_{\pi}(j)-2\right)+\sum_{j=1}^{N_{\pi N}}\left(\operatorname{dim} V_{\pi N}(j)-1\right)
$$

where $d_{\pi} \geq n$ is the chiral dimension of the pion operator. Because of parity invariance the vector operators $\mathcal{O}_{n, \pi}$ couple to 2 or more pions, whereas the axial vector operators $\widetilde{\mathcal{O}}_{n, \pi}^{A}(a)$ couple to 1 but not 2 pions. Starting at order $O\left(q^{n-k}\right)$ the form factors $E_{n, k+1}$ and $M_{n, k}$ thus receive corrections from the one-loop graphs shown in Fig. 2a and b. For isotriplet pion operators $n$ is odd due to charge conjugation invariance. Together with the time reversal invariance constraints on the nucleon form factors, one thus finds that the corrections to $E_{n, n-1}^{I=1}$ start at $O\left(q^{2}\right)$ and those to $M_{n, n-1}^{I=1}$ at order $O(q)$, whereas for all other form factors $E_{n, k+1}^{I=1}$ and $M_{n, k}^{I=1}$ they are at least of order $O\left(q^{3}\right)$.

The axial vector operator $\widetilde{\mathcal{O}}_{n, \pi}^{A}(a)$ contributes to nucleon matrix elements starting with the tree level graph in Fig 2 c. The $n$ vectors $a^{\mu}$ in the operator are all contracted with derivatives acting on the pion field and hence with $\Delta_{\mu}$ after evaluation of the graph. The same is true for the corresponding one-loop graphs. One thus obtains only contributions to the form factor $\widetilde{M}_{n, n-1}$, starting at order $O\left(q^{-2}\right)$ for the tree graph. With two loops one has graphs where three pions couple to the operator on one side and to the nucleon line on the other. Such graphs can contribute to other form factors, but only starting at order $O\left(q^{4}\right)$.

\section{Results for chiral-even isotriplet form factors}

\subsection{Relevant operators and graphs}

With the method outlined in Section 4.1 one finds vector operators

$$
\begin{aligned}
\mathcal{O}_{n, k+1,-1}^{A} & =\widetilde{E}_{n, k}^{I(0)}(i a \partial)^{k} \bar{N}_{v}(a S) \tau_{e-}^{A} N_{v}+\ldots, \\
\mathcal{O}_{n, k+1,0}^{A} & =\frac{1}{2} E_{n, k+1}^{I(0)}(i a \partial)^{k+1} \bar{N}_{v} \tau_{e+}^{A} N_{v}-\frac{1}{2} M_{n, k}^{I(0)}(i a \partial)^{k} i \partial_{\mu} \bar{N}_{v}\left[(a S), S^{\mu}\right] \tau_{e+}^{A} N_{v}+\ldots, \\
\mathcal{O}_{n, k+1,1}^{A} & =\frac{1}{4} \widetilde{M}_{n, k}^{I(0)}(i a \partial)^{k+1} i \partial_{\mu} \bar{N}_{v} S^{\mu} \tau_{e-}^{A} N_{v}+\ldots,
\end{aligned}
$$

where the ... stand for operators which have fewer total derivatives and as in Section 2 the isospin index $I=0$ belongs to $A=0$ and $I=1$ to $A=1,2,3$. The axial vector operators are simply obtained 
by interchanging $\tau_{e+}^{A}$ and $\tau_{e-}^{A}$,

$$
\begin{aligned}
\widetilde{\mathcal{O}}_{n, k+1,-1}^{A} & =\widetilde{E}_{n, k}^{I(0)}(i a \partial)^{k} \bar{N}_{v}(a S) \tau_{e+}^{A} N_{v}+\ldots, \\
\widetilde{\mathcal{O}}_{n, k+1,0}^{A} & =\frac{1}{2} E_{n, k+1}^{I(0)}(i a \partial)^{k+1} \bar{N}_{v} \tau_{e-}^{A} N_{v}-\frac{1}{2} M_{n, k}^{I(0)}(i a \partial)^{k} i \partial_{\mu} \bar{N}_{v}\left[(a S), S^{\mu}\right] \tau_{e-}^{A} N_{v}+\ldots, \\
\widetilde{\mathcal{O}}_{n, k+1,1}^{A} & =\frac{1}{4} \widetilde{M}_{n, k}^{I(0)}(i a \partial)^{k+1} i \partial_{\mu} \bar{N}_{v} S^{\mu} \tau_{e+}^{A} N_{v}+\ldots
\end{aligned}
$$

Using the rules (34) and the decomposition (8) the coefficients in (38) and (39) are easily identified as the tree-level contributions to the form factors $\widetilde{E}_{n, k}^{I}(t), E_{n, k+1}^{I}(t), M_{n, k}^{I}$ and $\widetilde{M}_{n, k}^{I}$ at order $O\left(q^{0}\right)$. Time reversal invariance implies that $E_{n, k+1}^{I(0)}$ is only nonzero for odd $k$, whereas the other coefficients $M_{n, k}^{I(0)}, \widetilde{E}_{n, k}^{I(0)}, \widetilde{M}_{n, k}^{I(0)}$ are only nonzero for even $k$. For $A=0$ we recover the isosinglet operators constructed in [13].

According to (29) insertions of an isotriplet operator with $\tau_{e-}^{a}$ require at least one pion line in the graph and hence do not contribute to nucleon matrix elements at tree level. They appear however in the one-loop graph shown in Fig. 11. When the pion-nucleon vertex in this graph is taken at LO one finds zero, because the loop integral is of the form

$$
\int d^{4-2 \epsilon} l \frac{(S l)}{(l v+w+i 0)\left(l^{2}-m^{2}+i 0\right)},
$$

whose numerator is proportional to $(S v)=0$ after the integration. Calculating the same graph to the next order, one finds that the contribution from the NLO pion-nucleon vertex cancels the one with the LO pion-nucleon vertex and an NLO nucleon propagator correction. This holds true for all operators with $\tau_{e-}^{a}$ in (38) and (39) and only requires that the operators does not introduce any dependence on the loop momentum $l_{\mu}$ via $\overleftrightarrow{\nabla}_{\mu}$ or $u_{\mu}$.

The operators with $\tau_{e+}^{a}$ contribute at tree level and via the loop graphs in Fig. 1a and b. They are constructed such that after the replacement $\partial_{\mu} \rightarrow i \Delta_{\mu}$ they match the structure of the terms in the form factor decomposition (8). That structure can be changed in loop graphs only when the spin vectors in the operator insertion are multiplied by spin vectors from pion-nucleon vertices. This is not the case for the graph in Fig. 1b, which originates from the two-pion term in the expansion (29) of $\tau_{e+}^{a}$ and thus reproduces the spin structure of the operator. Let us show that it is not the case either for the graph in Fig. 1 1 a with LO pion-nucleon vertices. The numerator of the corresponding loop integral has the form $(S l) \mathcal{O}(S l)$, where $\mathcal{O}$ contains zero, one or two vectors $S_{\mu}$ and represents the spin structure of the operator. The loop integration turns a tensor $l_{\mu_{1}} \ldots l_{\mu_{j}}$ into a combination of $v_{\mu}$ and $g_{\lambda \mu}$ and thus $(S l) \mathcal{O}(S l)$ into $S^{\rho} \mathcal{O} S_{\rho}$. This preserves the spin structure of $\mathcal{O}$ because

$$
S^{\rho} S_{\rho}=\frac{1}{4}(1-d), \quad S^{\rho} S^{\alpha} S_{\rho}=\frac{1}{4}(d-3) S^{\alpha}, \quad S^{\rho}\left[S^{\alpha}, S^{\beta}\right] S_{\rho}=\frac{1}{4}(5-d)\left[S^{\alpha}, S^{\beta}\right]
$$

in $d$ dimensions. We also need graphs with one LO and one NLO pion-nucleon vertex, or with two LO vertices and an NLO nucleon propagator correction. Restricting ourselves to the terms producing the required factors of $\Delta_{\mu}$ as discussed in Section 4.2, we obtain numerators $(l v)(S \Delta) \mathcal{O}(S l)$ or $(l \Delta)(S l) \mathcal{O}(S l)$, which give zero after loop integration.

In summary, the insertion of an operator from (38) or (39) into the graphs discussed so far either gives zero or contributes only to the same form factor for which it already provides the leading-order tree-level result. This is however not true for the graph in Fig 1 1 a with one NNLO or two NLO pion-nucleon interactions. In this case one obtains terms $(S \Delta) \mathcal{O}(S \Delta)$ after loop integration, which do change the spin structure of $\mathcal{O}$. 


\subsection{Vector form factors}

From Table 1 it follows that $E_{n, k+1}^{I}$ and $M_{n, k}^{I}$ can receive contributions from one-loop graphs with insertion of operators $\mathcal{O}_{n, l+1, i-1}$ with $l \geq k$ and $i \geq 0$. With the additional condition (36) required to produce enough factors of $\Delta_{\mu}$, we find that the form factors receive corrections of order $O\left(q^{2}\right)$ from graphs with LO pion-nucleon vertices and insertion of the operator $\mathcal{O}_{n, k+1,0}$, which already gives the tree-level contributions at order $O\left(q^{0}\right)$. By power counting one could also have order $O\left(q^{2}\right)$ contributions from graphs with insertion of $\mathcal{O}_{n, k+2,-1}$ or $\mathcal{O}_{n, k+1,-1}$ and pion-nucleon interactions at LO or NLO, respectively, but these vanish because the relevant operators come with $\tau_{e-}^{A}$. The one-loop corrections from pion-nucleon operators to $E_{n, k}^{I=1}(t)$ and $M_{n, k}^{I=1}(t)$ are then found to be

$$
\begin{aligned}
& E_{n, k}^{I=1(0)}\left(1-\frac{m^{2}}{(4 \pi F)^{2}}\left[\left(3 g_{A}^{2}+1\right) \log \frac{m^{2}}{\mu^{2}}+2 g_{A}^{2}\right]\right)+O\left(q^{3}\right), \\
& M_{n, k}^{I=1(0)}\left(1-\frac{m^{2}}{(4 \pi F)^{2}}\left[\left(2 g_{A}^{2}+1\right) \log \frac{m^{2}}{\mu^{2}}+2 g_{A}^{2}\right]\right)+O\left(q^{3}\right),
\end{aligned}
$$

respectively, where we have replaced the bare axial coupling $g_{0}$ by its physical value $g_{A}$ as is permissible within the precision of our result. Likewise, we could replace the bare pion decay constant $F$ and bare pion mass $m$ by their physical values $F_{\pi}$ and $m_{\pi}$ (we refrain from doing so for ease of notation). The contributions with $g_{A}^{2}$ in (42) are due to the graph in Fig. 19 and the nucleon wave function renormalization, and the contributions without $g_{A}^{2}$ come from the tadpole graph in Fig. 1b. As in 13] we use the renormalization scheme of [22], subtracting $1 / \epsilon+\log (4 \pi)+\psi(2)$ for each $1 / \epsilon$ pole in $4-2 \epsilon$ dimensions.

The form factors $E_{n, k+1}^{I}(t)$ and $M_{n, k}^{I}$ also receive chiral corrections from loop graphs with pion operator insertions. In the notation of [12] the isotriplet operators with lowest chiral dimension are 2

$$
\begin{aligned}
\mathcal{O}_{n, \pi}^{a}(a)= & 2 \tilde{b}_{n, n-1}(i a \partial)^{n-1}\left(a V^{a}\right) \\
& +2 i F^{2} \epsilon^{a b c} \sum_{\substack{k=0 \\
\text { even }}}^{n-3} \tilde{b}_{n, k}(i a \partial)^{k}\left[\left(a L^{b}\right)(2 i a \overleftrightarrow{\partial})^{n-k-2}\left(a L^{c}\right)+\left(a R^{b}\right)(2 i a \overleftrightarrow{\partial})^{n-k-2}\left(a R^{c}\right)\right]
\end{aligned}
$$

where

$$
V_{\mu}^{a}=-\frac{1}{2} i F^{2}\left(L_{\mu}^{a}+R_{\mu}^{a}\right), \quad L_{\mu}^{a} \tau^{a}=U^{\dagger} \partial_{\mu} U, \quad R_{\mu}^{a} \tau^{a}=U \partial_{\mu} U^{\dagger}
$$

To extract the terms coupling to two pions we use the expansion $L_{\mu}^{a}=i \partial_{\mu} \pi^{a} / F+i \epsilon^{a b c} \pi^{b} \partial_{\mu} \pi^{c} / F^{2}+$ $O\left(\pi^{3}\right)$ and its analog for $R_{\mu}^{a}$, obtained by changing the sign of the pion field 3 and obtain

$$
\begin{aligned}
\mathcal{O}_{n, \pi}^{a}(a)= & -2 i \epsilon^{a b c}\left\{\tilde{b}_{n, n-1}(i a \partial)^{n-1} \pi^{b}\left(i a \partial \pi^{c}\right)-2 \sum_{\substack{k=0 \\
\text { even }}}^{n-3} \tilde{b}_{n, k}(i a \partial)^{k}\left[\left(i a \partial \pi^{b}\right)(2 i a \overleftrightarrow{\partial})^{n-k-2}\left(i a \partial \pi^{c}\right)\right]\right\} \\
& +O\left(\pi^{4}\right)
\end{aligned}
$$

Using the relations

$$
4\left(i a \partial \pi^{b}\right)\left(i a \partial \pi^{c}\right)=(i a \partial)^{2} \pi^{b} \pi^{c}-\pi^{b}(2 i a \overleftrightarrow{\partial})^{2} \pi^{c}, \quad 2 \epsilon^{a b c} \pi^{b}\left(i a \partial \pi^{c}\right)=\epsilon^{a b c} \pi^{b}(2 i a \overleftrightarrow{\partial}) \pi^{c}
$$

\footnotetext{
${ }^{2}$ The normalization of the twist-two operators (2) used here agrees with the one in [13] and differs from that in 12 by a factor of 2 . The coefficients $\tilde{b}_{n, k}$ have the same normalization here and in 12 .

${ }^{3}$ We note that the sign of the term with $\epsilon^{a b c}$ in eq. (32) of [12] is incorrect.
} 
we can rewrite this as

$$
\mathcal{O}_{n, \pi}^{a}(a)=-i \epsilon^{a b c} \sum_{\substack{k=0 \\ \text { even }}}^{n-1} A_{n, k}^{\pi(0)}(i a \partial)^{k}\left[\pi^{b}(i a \overleftrightarrow{\partial})^{n-k} \pi^{c}\right]+O\left(\pi^{4}\right),
$$

where $A_{n, k}^{\pi(0)}=2^{n-k}\left(\tilde{b}_{n, k}-\tilde{b}_{n, k-2}\right)$ with $\tilde{b}_{n,-2}=0$. The coefficients $A_{n, k}^{\pi(0)}$ represent the chiral limit of the form factors $A_{n, k}^{\pi}(t)$ which parameterize the moments of the pion GPD as [12]

$$
\int_{-1}^{1} d x x^{n-1} H_{\pi}^{I}(x, \xi, t)=\sum_{\substack{k=0 \\ \text { even }}}^{n}(2 \xi)^{k} A_{n, k}^{\pi}(t),
$$

where in terms of quark flavors in a $\pi^{+}$one has $H_{\pi}^{I=0}=H_{\pi}^{u}+H_{\pi}^{d}$ and $H_{\pi}^{I=1}=H_{\pi}^{u}-H_{\pi}^{d}$. Because of isospin and charge conjugation symmetry one has $I=1$ for odd $n$ and $I=0$ for even $n$ and therefore can omit the isospin index $I$ in $A_{n, k}^{\pi}$.

As discussed after (37), the graphs in Fig. 22a and b with insertion of $\mathcal{O}_{n, \pi}^{a}(a)$ give rise to corrections which start at order $O\left(q^{2}\right)$ for $E_{n, n-1}^{I=1}$ and at order $O(q)$ for $M_{n, n-1}^{I=1}$. Together with (42) and with terms due to tree level operator insertions, the complete results to order $O\left(q^{2}\right)$ read

$$
\begin{aligned}
E_{n, k}^{I=1}(t)= & E_{n, k}^{I=1(0)}\left(1-\frac{m^{2}}{(4 \pi F)^{2}}\left[\left(3 g_{A}^{2}+1\right) \log \frac{m^{2}}{\mu^{2}}+2 g_{A}^{2}\right]\right) \\
& +\delta_{k, n-1} E_{n, n-1}^{I=1(2, \pi)}(t)+E_{n, k}^{I=1(2, m)} m^{2}+E_{n, k}^{I=1(2, t)} t+O\left(q^{3}\right), \\
M_{n, k}^{I=1}(t)= & M_{n, k}^{I=1(0)}\left(1-\frac{m^{2}}{(4 \pi F)^{2}}\left[\left(2 g_{A}^{2}+1\right) \log \frac{m^{2}}{\mu^{2}}+2 g_{A}^{2}\right]\right) \\
& +\delta_{k, n-1}\left[M_{n, n-1}^{I=1(1, \pi)}(t)+M_{n, n-1}^{I=1(2, \pi)}(t)\right]+M_{n, k}^{I=1(2, m)} m^{2}+M_{n, k}^{I=1(2, t)} t+O\left(q^{3}\right),
\end{aligned}
$$

where the contributions

$$
\begin{aligned}
E_{n, n-1}^{I=1(2, \pi)}(t)= & \frac{1}{2(4 \pi F)^{2}} \sum_{\substack{j=1 \\
\text { odd }}}^{n} 2^{-j} j A_{n, n-j}^{\pi(0)}\left\{4 g_{A}^{2} m^{2} \log \frac{m^{2}}{\mu^{2}}\right. \\
& \left.+\int_{-1}^{1} d \eta \eta^{j-1}\left[g_{A}^{2}\left(2 m^{2}-t\right)\left(\log \frac{m^{2}(\eta)}{\mu^{2}}+1\right)-\left(g_{A}^{2}-1\right) m^{2}(\eta) \log \frac{m^{2}(\eta)}{\mu^{2}}\right]\right\} \\
M_{n, n-1}^{I=1(1, \pi)}(t)= & -\frac{2 \pi M g_{A}^{2}}{(4 \pi F)^{2}} \sum_{j=1}^{n} 2^{-j} j A_{n, n-j}^{\pi(0)} \int_{-1}^{1} d \eta \eta^{j-1} m(\eta)
\end{aligned}
$$

with

$$
m^{2}(\eta)=m^{2}-\frac{t}{4}\left(1-\eta^{2}\right)
$$

are due to graphs with pion operator insertions and LO pion-nucleon vertices. The order $O\left(q^{2}\right)$ correction

$$
\begin{aligned}
& M_{n, n-1}^{I=1(2, \pi)}(t)=-\frac{1}{2(4 \pi F)^{2}} \sum_{j=1}^{n} 2^{-j} j A_{n, n-j}^{\pi(0)}\left\{4 g_{A}^{2} m^{2} \log \frac{m^{2}}{\mu^{2}}\right. \\
& \left.\quad+\int_{-1}^{1} d \eta \eta^{j-1}\left[g_{A}^{2}\left(2 m^{2}-t\right)\left(\log \frac{m^{2}(\eta)}{\mu^{2}}+1\right)+\left(g_{A}^{2}-1-4 M c_{4}\right) m^{2}(\eta) \log \frac{m^{2}(\eta)}{\mu^{2}}\right]\right\}
\end{aligned}
$$


is due to graphs with one NLO pion-nucleon vertex or nucleon propagator correction, as well as graphs with LO vertices and the subleading part $w v^{\mu}$ of the residual nucleon momenta, cf. the discussion after (33). The terms proportional to $g_{A}^{2}$ in (50) and (52) are due to the graph in Fig. 2al, and the other terms to the graph in Fig. 2b. Our expressions (49) and (50) agree with the results in [14], where the order $O\left(q^{2}\right)$ corrections to $E_{n, n-1}^{I=1}$ and the order $O(q)$ corrections to $M_{n, n-1}^{I=1}$ are given.

\subsection{Axial form factors}

Using Table 1 and the condition (36), one readily finds that the chiral corrections of order $O\left(q^{2}\right)$ to the form factor $\tilde{E}_{n, k}^{I}$ are obtained from graphs with LO vertices and insertion of the operator $\widetilde{\mathcal{O}}_{n, k+1,-1}$, which already gives the tree-level contributions at order $O\left(q^{0}\right)$. Together with higher-order tree level insertions we get

$$
\begin{aligned}
\widetilde{E}_{n, k}^{I=1}(t)= & \widetilde{E}_{n, k}^{I=1(0)}\left(1-\frac{m^{2}}{(4 \pi F)^{2}}\left[\left(2 g_{A}^{2}+1\right) \log \frac{m^{2}}{\mu^{2}}+g_{A}^{2}\right]\right) \\
& +\widetilde{E}_{n, k}^{I=1(2, m)} m^{2}+\widetilde{E}_{n, k}^{I=1(2, t)} t+O\left(q^{3}\right),
\end{aligned}
$$

in agreement with [14]. The discussion of contributions to $\widetilde{M}_{n, k}^{I}$ is more involved; for the isotriplet case it proceeds in close analogy to the isosinglet case analyzed in [13. According to Table 1 and the condition (36), one obtains order $O\left(q^{2}\right)$ corrections from graphs with insertion of $\widetilde{\mathcal{O}}_{n, k+1,1}$ and LO vertices. Further corrections are due to graphs with insertion of $\widetilde{\mathcal{O}}_{n, k+1,-1}$ and two NLO pion-nucleon vertices or nucleon propagator corrections, or with one NNLO pion-nucleon vertex generated by (35). Graphs with the same operator insertion and two loops or one loop and a pion propagator correction from $\mathcal{L}_{\pi}^{(4)}$ could contribute by power counting but do not produce the required factors of $\Delta_{\mu}$ (see [13]). Graphs with insertion of $\widetilde{\mathcal{O}}_{n, k+2,0}$ or $\widetilde{\mathcal{O}}_{n, k+1,0}$ and pion-nucleon interactions at LO or NLO give zero because these operators involve $\tau_{e-}^{a}$, as discussed after (40). Graphs involving $\widetilde{\mathcal{O}}_{n, k+2,-1}$ and NLO pion-nucleon interactions do not contribute to $\widetilde{M}_{n, k}^{I}$ due to time reversal invariance, since the operator is only nonzero for odd $k$ and the form factor only for even $k$. Finally, graphs with insertion of $\widetilde{\mathcal{O}}_{n, k+3,-1}$ and LO pion-nucleon vertices contribute to $\widetilde{E}_{n, k+2}^{I}$ but not to $\widetilde{M}_{n, k}^{I}$ as discussed at the end of Section 5.1 .

Together with higher-order tree-level insertions, the one-loop graphs with $\widetilde{\mathcal{O}}_{n, k+1,1}$ or $\widetilde{\mathcal{O}}_{n, k+1,-1}$ thus give the full result at order $O\left(q^{2}\right)$ for the form factors with $k<n-1$,

$$
\begin{aligned}
\widetilde{M}_{n, k}^{I=1}(t)= & \widetilde{M}_{n, k}^{I=1(0)}\left(1-\frac{m^{2}}{(4 \pi F)^{2}}\left[\left(2 g_{A}^{2}+1\right) \log \frac{m^{2}}{\mu^{2}}+g_{A}^{2}\right]\right) \\
& +\widetilde{E}_{n, k}^{I=1(0)} \frac{m^{2} g_{A}^{2}}{3(4 \pi F)^{2}} \log \frac{m^{2}}{\mu^{2}}+\widetilde{M}_{n, k}^{I=1(2, m)} m^{2}+\widetilde{M}_{n, k}^{I=1(2, t)} t+O\left(q^{3}\right) .
\end{aligned}
$$

The form factors $\widetilde{M}_{n, n-1}^{I=1}$ require a separate discussion because they receive a contribution starting at order $O\left(q^{-2}\right)$ from the one-pion exchange graph in Fig. 2 2 c, as discussed at the end of Section 4.2 , The relevant operator is given by

$$
\widetilde{\mathcal{O}}_{n, \pi}^{a}(a)=2 \tilde{b}_{n, n-1}(i a \partial)^{n-1}\left(a A^{a}\right)\left[1+\left(\frac{l_{4}^{r}}{2 F^{2}}+\tilde{c}_{n}\right) \operatorname{Tr} \chi_{+}\right]+O\left(q^{n+4}\right)
$$

with odd $n$ and

$$
A_{\mu}^{a}=-\frac{1}{2} i F^{2}\left(R_{\mu}^{a}-L_{\mu}^{a}\right),
$$


where $\tilde{b}_{n, n-1}$ is the same as in (43) because of parity invariance. $l_{4}^{r}$ is the renormalized low-energy constant from the pion Lagrangian (17) and appears in the expression of the axial current,

$$
\frac{1}{2} \bar{q} \gamma_{\mu} \gamma_{5} \tau^{a} q \cong A_{\mu}^{a}\left[1+\frac{l_{4}^{r}}{2 F^{2}} \operatorname{Tr} \chi_{+}\right]+O\left(q^{5}\right)
$$

so that $\tilde{b}_{1,0}=1$ and $\tilde{c}_{1}=0$. One can readily derive (57) by coupling the Lagrangian to an external isovector axial field $a_{\mu}$ as usual [19], which implies $u_{\mu}=i\left(u^{\dagger} \partial_{\mu} u-u \partial u^{\dagger}\right)+u^{\dagger} a_{\mu} u+u a_{\mu} u^{\dagger}$. As an aside we note that the correction with $l_{4}^{r}$ in the axial current (57) would be different if one used the pion Lagrangian from [22, where the term involving this low-energy constant reads

$$
\frac{l_{4}}{4} \operatorname{Tr}\left[\left(\partial_{\mu} \chi^{\dagger}\right)\left(\partial^{\mu} U\right)+\left(\partial_{\mu} \chi\right)\left(\partial^{\mu} U^{\dagger}\right)\right]=\frac{l_{4}}{8}\left\{\operatorname{Tr} \chi_{+} \operatorname{Tr}\left(u_{\mu} u^{\mu}\right)+2 i \operatorname{Tr}\left[\nabla_{\mu}, \chi_{-}\right] u^{\mu}\right\} .
$$

In the present work we follow Ref. [18] and use the Lagrangian (17) from [17]. It differs from (58) by total derivative terms and terms that vanish by the equation of motion. With the full Lagrangian given by $\mathcal{L}_{\pi}+\mathcal{L}_{\pi N}$ the equation of motion for the pion field involves terms bilinear in the nucleon field 4 so that a change of $\mathcal{L}_{\pi}$ using the equation of motion induces a corresponding change in $\mathcal{L}_{\pi N}$. We also refer to the discussion in [23].

The coefficients $\tilde{b}_{n, n-1}$ and $\tilde{c}_{n}$ in (55) appear in the moments of the twist-two pion distribution amplitude $\phi_{\pi}(x)$, which are defined by

$$
\int \frac{d \eta}{2 \pi} e^{i x \eta(a p)}\left\langle\pi^{b}(p)\left|\bar{q}(-\eta a) \not \gamma \gamma_{5} \tau^{a} q(\eta a)\right| 0\right\rangle=-i \delta^{a b} F_{\pi} \phi_{\pi}(x)
$$

and

$$
B_{n}^{\pi}=2^{-n} \int_{-1}^{1} d x x^{n-1} \phi_{\pi}(x)
$$

so that

$$
\left\langle\pi^{b}(p)\left|\widetilde{\mathcal{O}}_{n, \pi}^{a}(a)\right| 0\right\rangle=-2 i \delta^{a b}(a p)^{n} F_{\pi} B_{n}^{\pi} .
$$

Calculating the leading chiral correction to this matrix element one finds that $l_{4}^{r}$ only appears in the expression of the pion decay constant,

$$
F_{\pi}=F\left(1-\frac{m^{2}}{(4 \pi F)^{2}} \log \frac{m^{2}}{\mu^{2}}+\frac{m^{2}}{F^{2}} l_{4}^{r}(\mu)+O\left(m^{4}\right)\right),
$$

and $\tilde{c}_{n}$ only in the correction to the moments

$$
B_{n}^{\pi}=\tilde{b}_{n, n-1}\left(1+4 m^{2} \tilde{c}_{n}\right)+O\left(m^{4}\right) .
$$

The definition of the pion decay constant implies $B_{1}^{\pi}=1$ to all orders in the chiral expansion. be

Returning to the nucleon form factors $\widetilde{M}_{n, n-1}^{I=1}$, one readily finds their lowest-order contribution to

$$
\widetilde{M}_{n, n-1}^{I=1(-2)}(t)=B_{n}^{\pi(0)} \frac{4 M^{2} g_{0}}{m^{2}-t}
$$

with $B_{n}^{\pi(0)}=\tilde{b}_{n, n-1}$. The leading corrections to this come from a number of higher-order operator insertions and loop graphs. A tadpole insertion in the pion line of the graph in Fig. 2 c and the

\footnotetext{
${ }^{4}$ With an arbitrary matrix $X$ in isospin space and $\tilde{X}=X-\frac{1}{2} \operatorname{Tr} X$, the leading-order equation of motion reads $2 i \operatorname{Tr}\left[\nabla_{\mu}, u^{\mu}\right] X+\operatorname{Tr} \chi_{-} \tilde{X}=F^{-2} \bar{N}_{v}\left[(v u)+4 i g_{0}(S \nabla), \tilde{X}\right] N_{v}-4 i g_{0} F^{-2} \partial^{\mu}\left(\bar{N}_{v} S_{\mu} \tilde{X} N_{v}\right)$.
} 
propagator correction from the $l_{3}$ term in the pion Lagrangian (17) result in a shift $m^{2} \rightarrow m_{\pi}^{2}$ in (64), where

$$
m_{\pi}^{2}=m^{2}\left(1+\frac{m^{2}}{2(4 \pi F)^{2}} \log \frac{m^{2}}{\mu^{2}}+\frac{2 m^{2}}{F^{2}} l_{3}^{r}(\mu)+O\left(m^{4}\right)\right)
$$

The pion propagator correction from the $l_{4}$ term in (17) cancels against the $l_{4}$ term from the operator (55). A tadpole insertion at the operator vertex gives a chiral logarithm as in (62). Further corrections are due to loop corrections to the pion-nucleon vertex, to tree-level insertions from $\mathcal{L}_{\pi N}^{(3)}$, and to the factors $\mathcal{N}^{2}$ and $Z_{N}$ in the matching formula (21). Together with the tree-level insertion of the pionnucleon operator $\widetilde{\mathcal{O}}_{n, n, 1}^{a}$ from (39) we obtain

$$
\begin{aligned}
\widetilde{M}_{n, n-1}^{I=1}(t)=B_{n}^{\pi(0)} \frac{4 M^{2} g_{0}}{m_{\pi}^{2}-t} & \left(1-\frac{m^{2}}{(4 \pi F)^{2}}\left[\left(2 g_{0}^{2}+1\right) \log \frac{m^{2}}{\mu^{2}}+g_{0}^{2}\right]\right. \\
& \left.+2 m^{2} g_{0}^{-1}\left(2 d_{16}^{r}-d_{18}\right)-8 m^{2} d_{28}^{r}+4 m^{2} \tilde{c}_{n}\right)+\widetilde{M}_{n, n-1}^{I=1(0)}+O(q)
\end{aligned}
$$

with the low-energy constants $d_{16}^{r}(\mu), d_{18}$ and $d_{28}^{r}(\mu)$ from [21]. To make the pion mass dependence fully explicit, one should replace $M^{2}=M_{0}^{2}-8 m^{2} M_{0} c_{1}+O\left(m^{3}\right)$. Conversely, we can use (63) and the one-loop expression

$$
g_{A}=g_{0}\left(1-\frac{m^{2}}{(4 \pi F)^{2}}\left[\left(2 g_{0}^{2}+1\right) \log \frac{m^{2}}{\mu^{2}}+g_{0}^{2}\right]+4 m^{2} g_{0}^{-1} d_{16}^{r}-8 m^{2} d_{28}^{r}\right)+O\left(m^{3}\right)
$$

of the axial coupling (see e.g. 24]) to rewrite the result as

$$
\widetilde{M}_{n, n-1}^{I=1}(t)=B_{n}^{\pi} \frac{4 M^{2} g_{A}}{m_{\pi}^{2}-t}\left(1-2 m_{\pi}^{2} g_{A}^{-1} d_{18}\right)+\widetilde{M}_{n, n-1}^{I=1(0)}+O(q)
$$

in terms of the physical quantities $B_{n}^{\pi}, g_{A}, M, m_{\pi}$ and the low-energy constants $d_{18}$ and $\widetilde{M}_{n, n-1}^{I=1(0)}$. With the transformation (10) and the definitions (5) and (60) of moments one finds

$$
\widetilde{E}^{I=1}(x, \xi, t)=\frac{\theta(|x|<\xi)}{2 \xi} \phi_{\pi}\left(\frac{x}{\xi}\right) \frac{4 M^{2} g_{A}}{m_{\pi}^{2}-t}\left(1-2 m_{\pi}^{2} g_{A}^{-1} d_{18}\right)+\widetilde{E}^{I=1(0)}(x, \xi)+O(q),
$$

which generalizes the well-known relation from [25, 26] to next-to-leading order in the chiral expansion. For $n=1$ our result (68) is consistent with the one for the pseudoscalar form factor in [18, 27]. We also agree with the result of Ref. [14] if in their eq. (66) one adds a term $\delta_{m, 2 k} \frac{2}{3} g_{A} M^{2}\left\langle r_{A}^{2}\right\rangle\left\langle z^{2 k}\right\rangle_{\pi} / 2^{2 k}$.

\section{Chiral-odd generalized parton distributions}

In this section we consider the general parton distributions associated with chiral-odd operators. As in the previous sections we restrict ourselves to the twist-two sector. The relevant GPDs of the nucleon are defined by

$$
\begin{aligned}
\int \frac{d \eta}{4 \pi} e^{i x \eta(a P)}\left\langle N_{i}\left(p^{\prime}\right)\left|\bar{q}\left(-\frac{1}{2} \eta a\right) b_{\lambda} a_{\mu} i \sigma^{\lambda \mu} \tau^{A} q\left(\frac{1}{2} \eta a\right)\right| N_{j}(p)\right\rangle & \\
=\tau_{i j}^{A} \frac{1}{2 a P} b_{\lambda} a_{\mu} \bar{u}\left(p^{\prime}\right) & {\left[i \sigma^{\lambda \mu} H_{T}^{I}+\frac{\gamma^{\lambda} \Delta^{\mu}-\Delta^{\lambda} \gamma^{\mu}}{2 M} \bar{E}_{T}^{I}\right.} \\
& \left.-\frac{i \sigma^{\lambda \nu} \Delta^{\mu} \Delta_{\nu}-i \sigma^{\mu \nu} \Delta^{\lambda} \Delta_{\nu}}{2 M^{2}} \widetilde{H}_{T}^{I}+\frac{\gamma^{\lambda} P^{\mu}-P^{\lambda} \gamma^{\mu}}{M} \widetilde{E}_{T}^{I}\right] u(p) .
\end{aligned}
$$

\footnotetext{
${ }^{5}$ We have traded the distribution $E_{T}$ in the original decomposition [28] for the combination $\bar{E}_{T}=E_{T}+2 \widetilde{H}_{T}$, which naturally appears when representing the distributions at $\xi=0$ in terms of densities in the impact parameter plane 8 .
} 
In addition to the light-like vector $a$ we have introduced a vector $b$ satisfying $a b=0$, and for brevity we have suppressed the arguments $x, \xi, t$ of the distributions 6 Their Mellin moments are related to the matrix elements of the chiral-odd twist-two operators

$$
\mathcal{O}_{T \lambda \mu_{1} \mu_{2} \ldots \mu_{n}}^{A}=\underset{\lambda \mu_{1} \ldots \mu_{n}}{\mathrm{~T}} \underset{\lambda \mu_{1}}{\mathrm{~A}} \underset{\mu_{1} \ldots \mu_{n}}{\mathrm{~S}} \bar{q} \sigma_{\lambda \mu_{1}} i \stackrel{\leftrightarrow}{D}_{\mu_{2}} \ldots i \overleftrightarrow{D}_{\mu_{n}} \tau^{A} q
$$

where $\mathrm{T}$ and $\mathrm{S}$ are defined as in Section 2 and where $\mathrm{A}$ denotes antisymmetrization, $\mathrm{A}_{\lambda \mu} t^{\lambda \mu}=$ $\frac{1}{2}\left(t^{\lambda \mu}-t^{\mu \lambda}\right)$. These operations are conveniently implemented by contraction with the auxiliary vectors $a$ and $b$, given that for any tensor $t^{\lambda \mu_{1} \ldots \mu_{n}}$ satisfying $t^{\lambda \mu_{1} \mu_{2} \ldots \mu_{n}}=-t^{\mu_{1} \lambda \mu_{2} \ldots \mu_{n}}$ one has

$$
\begin{aligned}
& b_{\lambda} a_{\mu_{1}} a_{\mu 2} \ldots a_{\mu_{n}} \underset{\lambda \mu_{1} \ldots \mu_{n}}{\mathrm{~T}} \underset{\lambda \mu_{1}}{\mathrm{~A}} \underset{\mu_{1} \ldots \mu_{n}}{\mathrm{~S}} t^{\lambda \mu_{1} \ldots \mu_{n}} \\
& =b_{\lambda} a_{\mu_{1}} a_{\mu 2} \ldots a_{\mu_{n}} \frac{1}{2 n}\left(t^{\lambda \mu_{1} \mu_{2} \ldots \mu_{n}}+\sum_{i=2}^{n} t^{\lambda \mu_{2} \ldots \mu_{i} \mu_{1} \mu_{i+1} \ldots \mu_{n}}-t^{\mu_{1} \lambda \mu_{2} \ldots \mu_{n}}-\sum_{i=2}^{n} t^{\mu_{1} \mu_{2} \ldots \mu_{i} \lambda \mu_{i+1} \ldots \mu_{n}}\right) \\
& =\frac{n+1}{2 n} b_{\lambda} a_{\mu_{1}} a_{\mu_{2}} \ldots a_{\mu_{n}} t^{\lambda \mu_{1} \ldots \mu_{n}} .
\end{aligned}
$$

where symmetrization in $\mu_{2} \ldots \mu_{n}$ is guaranteed by contraction with identical vectors, and where trace subtraction terms are removed by the conditions $a^{2}=a b=0$. The $(n-1)$ terms of the second sum give zero due to the antisymmetry of $t$ in its first two indices. We therefore define the contracted operator

$$
\mathcal{O}_{T n}^{A}(a, b)=\frac{2 n}{n+1} b^{\lambda} a^{\mu_{1}} a^{\mu_{2}} \ldots a^{\mu_{n}} \mathcal{O}_{T \lambda \mu_{1} \mu_{2} \ldots \mu_{n}}^{A}=\bar{q} b^{\lambda} a^{\mu} i \sigma_{\lambda \mu}(i a \stackrel{\leftrightarrow}{D})^{n-1} \tau^{A} q
$$

whose nucleon matrix elements are parameterized by

$$
\begin{aligned}
\left\langle N_{i}\left(p^{\prime}\right)\left|\mathcal{O}_{T n}^{A}(a, b)\right| N_{j}(p)\right\rangle= & \tau_{i j}^{A} \sum_{k=0}^{n-1}(a P)^{n-k-1}(a \Delta)^{k} b_{\lambda} a_{\mu} \bar{u}\left(p^{\prime}\right)\left[i \sigma^{\lambda \mu} A_{T n, k}^{I}+\frac{\gamma^{\lambda} \Delta^{\mu}-\Delta^{\lambda} \gamma^{\mu}}{2 M} \bar{B}_{T n, k}^{I}\right. \\
& \left.-\frac{i \sigma^{\lambda \nu} \Delta^{\mu} \Delta_{\nu}-i \sigma^{\mu \nu} \Delta^{\lambda} \Delta_{\nu}}{2 M^{2}} \widetilde{A}_{T n, k}^{I}+\frac{\gamma^{\lambda} P^{\mu}-P^{\lambda} \gamma^{\mu}}{M} \widetilde{B}_{T n, k}^{I}\right] u(p) .
\end{aligned}
$$

The moments of the chiral-odd GPDs are then expressed as [29]

$$
\begin{aligned}
\int_{-1}^{1} d x x^{n-1} H_{T}(x, \xi, t) & =\sum_{\substack{k=0 \\
\text { even }}}^{n-1}(-2 \xi)^{k} A_{T n, k}(t), \quad \int_{-1}^{1} d x x^{n-1} \bar{E}_{T}(x, \xi, t)=\sum_{\substack{k=0 \\
\text { even }}}^{n-1}(-2 \xi)^{k} \bar{B}_{T n, k}(t), \\
\int_{-1}^{1} d x x^{n-1} \widetilde{H}_{T}(x, \xi, t) & =\sum_{\substack{k=0 \\
\text { even }}}^{n-1}(-2 \xi)^{k} \widetilde{A}_{T n, k}(t), \quad \int_{-1}^{1} d x x^{n-1} \widetilde{E}_{T}(x, \xi, t)=\sum_{\substack{k=1 \\
\text { odd }}}^{n-1}(-2 \xi)^{k} \widetilde{B}_{T n, k}(t),
\end{aligned}
$$

where we have omitted isospin indices $I$ in the distributions and form factors for ease of writing. The restrictions to even or odd $k$ for the form factors reflect that $H_{T}, \bar{E}_{T}$ and $\widetilde{H}_{T}$ are even in $\xi$ and $\widetilde{E}_{T}$ is odd in $\xi$ due of time reversal invariance [28].

Using the relations (9) in [13] and

$$
\bar{u}\left(p^{\prime}\right) i \sigma^{\lambda \mu} u(p)=\bar{u}_{v}\left(p^{\prime}\right)\left[2 \gamma\left[S^{\lambda}, S^{\mu}\right]+\frac{v^{\lambda} \Delta^{\mu}-v^{\mu} \Delta^{\lambda}}{2 M}+\frac{\left[S^{\lambda},(S \Delta)\right] \Delta^{\mu}-\left[S^{\mu},(S \Delta)\right] \Delta^{\lambda}}{2 M^{2}(1+\gamma)}\right] u_{v}(p)
$$

\footnotetext{
${ }^{6}$ Instead of contracting $\sigma^{\lambda \mu}$ with auxiliary vectors one often takes definite indices $\sigma^{i+}$, where $i=1,2$ denotes a transverse component and + the plus-component in light-cone coordinates, i.e. $\sigma^{i+}=\left(\sigma^{i 0}+\sigma^{i 3}\right) / \sqrt{2}$.
} 
one can rewrite the decomposition (74) in terms of the heavy-baryon spinors (7) and obtains

$$
\begin{aligned}
& \left\langle N_{i}\left(p^{\prime}\right)\left|\mathcal{O}_{T n}^{A}(a, b)\right| N_{j}(p)\right\rangle=\tau_{i j}^{A} \sum_{k=0}^{n-1}(M \gamma)^{n-k-1}(a v)^{n-k-1}(a \Delta)^{k} b_{\lambda} a_{\mu} \\
& \times \bar{u}_{v}\left(p^{\prime}\right)\left[2 \gamma\left[S^{\lambda}, S^{\mu}\right] \widetilde{E}_{T n, k}^{I}+\frac{\left[S^{\lambda},(S \Delta)\right] \Delta^{\mu}-\left[S^{\mu},(S \Delta)\right] \Delta^{\lambda}}{2 M^{2}} \widetilde{M}_{T n, k}^{I}\right. \\
& \left.\quad+\gamma \frac{\left[S^{\lambda},(S \Delta)\right] v^{\mu}-\left[S^{\mu},(S \Delta)\right] v^{\lambda}}{M} E_{T n, k}^{I}+\frac{v^{\lambda} \Delta^{\mu}-v^{\mu} \Delta^{\lambda}}{2 M} M_{T n, k}^{I}\right] u_{v}(p)
\end{aligned}
$$

with new form factors given by

$$
\begin{array}{ll}
\widetilde{E}_{T n, k}=A_{T n, k}, & \widetilde{M}_{T n, k}=(1+\gamma)^{-1} A_{T n, k}+\bar{B}_{T n, k}-2 \widetilde{A}_{T n, k} . \\
E_{T n, k}=\widetilde{B}_{T n, k}, & M_{T n, k}=A_{T n, k}+\bar{B}_{T n, k}-\frac{\Delta^{2}}{2 M^{2}} \widetilde{A}_{T n, k},
\end{array}
$$

or equivalently

$$
\begin{aligned}
\bar{B}_{T n, k} & =\frac{1}{\gamma^{2}}\left[M_{T n, k}-\frac{\Delta^{2}}{4 M^{2}} \widetilde{M}_{T n, k}-\gamma \widetilde{E}_{T n, k}\right], \\
\widetilde{A}_{T n, k} & =\frac{1}{2 \gamma^{2}}\left[M_{T n, k}-\widetilde{M}_{T n, k}-\frac{\gamma}{1+\gamma} \widetilde{E}_{T n, k}\right] .
\end{aligned}
$$

We finish this section by defining chiral-odd GPDs in the pion,

$$
\begin{aligned}
& \int \frac{d \eta}{4 \pi} e^{i x \eta(a P)}\left\langle\pi^{c}\left(p^{\prime}\right)\left|\bar{q}\left(-\frac{1}{2} \eta a\right) b_{\lambda} a_{\mu} i \sigma^{\lambda \mu} \tau^{A} q\left(\frac{1}{2} \eta a\right)\right| \pi^{b}(p)\right\rangle \\
& =\frac{1}{2} \operatorname{Tr}\left(\tau^{A} \tau^{b} \tau^{c}\right) \frac{1}{2 a P} b_{\lambda} a_{\mu} \frac{P^{\lambda} \Delta^{\mu}-\Delta^{\lambda} P^{\mu}}{m_{\pi}} E_{T \pi}^{I}(x, \xi, t),
\end{aligned}
$$

where as in the nucleon case, isospin $I=0$ corresponds to $A=0$ and $I=1$ to $A=1,2,3$. In terms of quark flavors in a $\pi^{+}$one has $E_{T \pi}^{I=0}=E_{T \pi}^{u}+E_{T, \pi}^{d}$ and $E_{T \pi}^{I=1}=E_{T \pi}^{u}-E_{T \pi}^{d}$, with the definition

$$
\begin{gathered}
\int \frac{d \eta}{4 \pi} e^{i x \eta(a P)}\left\langle\pi^{+}\left(p^{\prime}\right)\left|\bar{u}\left(-\frac{1}{2} \eta a\right) b_{\lambda} a_{\mu} i \sigma^{\lambda \mu} u\left(\frac{1}{2} \eta a\right)\right| \pi^{+}(p)\right\rangle \\
=\frac{1}{2 a P} b_{\lambda} a_{\mu} \frac{P^{\lambda} \Delta^{\mu}-\Delta^{\lambda} P^{\mu}}{m_{\pi}} E_{T \pi}^{u}(x, \xi, t)
\end{gathered}
$$

and its analog for $d$ quarks. For the local twist-two operators one has

$$
\left\langle\pi^{c}\left(p^{\prime}\right)\left|\mathcal{O}_{T n}^{A}(a, b)\right| \pi^{b}(p)\right\rangle=\frac{1}{2} \operatorname{Tr}\left(\tau^{A} \tau^{b} \tau^{c}\right) b_{\lambda} a_{\mu} \frac{P^{\lambda} \Delta^{\mu}-\Delta^{\lambda} P^{\mu}}{m_{\pi}} \sum_{\substack{k=0 \\ \text { even }}}^{n-1}(a P)^{n-k-1}(a \Delta)^{k} B_{T n, k}^{\pi}(t)
$$

with

$$
\int_{-1}^{1} d x x^{n-1} E_{T \pi}^{I}(x, \xi, t)=\sum_{\substack{k=0 \\ \text { even }}}^{n-1}(2 \xi)^{k} B_{T n, k}^{\pi}(t),
$$

where the restriction to even $k$ is a consequence of time reversal symmetry. Due to isospin and charge conjugation invariance, $n$ is even for $I=0$ and odd for $I=1$, so that we do not need an isospin label for $B_{n, k}^{T \pi}$. 


\section{$7 \quad$ Chiral-odd effective operators}

In this section we explain how to construct the operators in the effective theory that match the chiralodd quark operators (71), closely following the strategy used in Section 4. To this end we first match the operators

$$
\left(\mathcal{O}_{R L, n}^{\lambda \mu}(a)\right)_{i j}=\bar{q}_{j} i \sigma^{\lambda \mu} \frac{1+\gamma_{5}}{2}(i a \overleftrightarrow{D})^{n-1} q_{i}, \quad\left(\mathcal{O}_{L R, n}^{\lambda \mu}(a)\right)_{i j}=\bar{q}_{j} i \sigma^{\lambda \mu} \frac{1-\gamma_{5}}{2}(i a \overleftrightarrow{D})^{n-1} q_{i}
$$

with open isospin indices $i, j$, which involve quarks of definite chirality. The corresponding uncontracted operators $\frac{1}{2} \bar{q}_{j} i \sigma_{\lambda \mu_{1}}\left(1 \pm \gamma_{5}\right) i \overleftrightarrow{D}_{\mu_{2}} \ldots i \overleftrightarrow{D}_{\mu_{n}} q_{i}$ do not have definite twist, but according to (72) their twist-two part is readily projected out in $b_{\lambda} a_{\mu} \mathcal{O}_{R L, n}^{\lambda \mu}(a)$ and $b_{\lambda} a_{\mu} \mathcal{O}_{L R, n}^{\lambda \mu}(a)$. The operators (84) transform as

$$
\mathcal{O}_{R L, n}^{\lambda \mu}(a) \rightarrow V_{R} \mathcal{O}_{R L, n}^{\lambda \mu}(a) V_{L}^{\dagger} \quad \mathcal{O}_{L R, n}^{\lambda \mu}(a) \rightarrow V_{L} \mathcal{O}_{L R, n}^{\lambda \mu}(a) V_{R}^{\dagger}
$$

unter chiral rotations and are transformed into each other by parity. Because $\sigma^{\lambda \mu} \gamma_{5}=-\frac{1}{2} i \epsilon^{\lambda \mu}{ }_{\alpha \beta} \sigma^{\alpha \beta}$ they obey the duality relations

$$
\mathcal{O}_{R L, n}^{\lambda \mu}(a)=-\frac{i}{2} \epsilon^{\lambda \mu}{ }_{\alpha \beta} \mathcal{O}_{R L, n}^{\alpha \beta}(a) \quad \mathcal{O}_{L R, n}^{\lambda \mu}(a)=\frac{i}{2} \epsilon^{\lambda \mu}{ }_{\alpha \beta} \mathcal{O}_{L R, n}^{\alpha \beta}(a)
$$

The operators $\mathcal{O}_{T n}^{A}(a, b)$ from (173), which correspond to twist-two and to definite isospin, are obtained as

$$
\mathcal{O}_{T n}^{A}(a, b)=b_{\lambda} a_{\mu} Q_{n}^{A, \lambda \mu}(a), \quad Q_{n}^{A, \lambda \mu}(a)=\operatorname{Tr} \tau^{A}\left\{\mathcal{O}_{R L, n}^{\lambda \mu}(a)+\mathcal{O}_{L R, n}^{\lambda \mu}(a)\right\}
$$

They will involve the combinations

$$
\tau_{o \pm}^{A}=u^{\dagger} \tau^{A} u^{\dagger} \pm u \tau^{A} u
$$

whose expansion in pion fields reads

$$
\begin{array}{lll}
\tau_{o+}^{0}=2-\frac{1}{F^{2}} \pi^{a} \pi^{a}+O\left(\pi^{4}\right), & \tau_{o-}^{0}=-\frac{2 i}{F} \pi^{a} \tau^{a}+O\left(\pi^{3}\right), \\
\tau_{o+}^{a}=2 \tau^{a}-\frac{1}{F^{2}} \pi^{a} \pi^{b} \tau^{b}+O\left(\pi^{4}\right), & \tau_{o-}^{a}=-\frac{2 i}{F} \pi^{a}+O\left(\pi^{3}\right) .
\end{array}
$$

As for the chiral-even case discussed in Section 4.1, the operators which match (84) in the effective theory and contribute to nucleon matrix elements are either bilinear in the nucleon field or contain only pion operators. We treat the two cases in the following two subsections.

\subsection{Pion-nucleon operators}

The effective operators which are bilinear in the nucleon field and transform as (85) can be written in the form

$$
\left(\mathcal{O}_{R L, n}^{\lambda \mu}(a)\right)_{i j}=\left(\bar{N}_{v} \mathcal{O}_{1} u\right)_{j}\left(u \mathcal{O}_{2} N_{v}\right)_{i}, \quad\left(\mathcal{O}_{L R, n}^{\lambda \mu}(a)\right)_{i j}=\left(\bar{N}_{v} \mathcal{O}_{1}^{\prime} u^{\dagger}\right)_{j}\left(u^{\dagger} \mathcal{O}_{2}^{\prime} N_{v}\right)_{i}
$$

where $\mathcal{O}_{1}, \mathcal{O}_{2}$ involve the fields $u_{\mu}, \chi_{ \pm}$and covariant derivatives and transform like $u_{\mu}$ under chiral rotations. $\mathcal{O}_{1}^{\prime}$ and $\mathcal{O}_{2}^{\prime}$ are related to $\mathcal{O}_{1}$ and $\mathcal{O}_{2}$ by parity. One can rearrange the covariant derivatives in $\mathcal{O}_{R L, n}^{\lambda \mu}$ and $\mathcal{O}_{L R, n}^{\lambda \mu}$ such that they act either as total derivatives $\partial_{\mu}$ or in the antisymmetric form $\overleftrightarrow{\nabla}_{\mu}=\frac{1}{2}\left(\vec{\nabla}_{\mu}-\overleftarrow{\nabla}_{\mu}\right)$ 
Table 2: Overview of contributions to the chiral-odd form factors. As in Table 1 the restriction in the second column is due to time reversal invariance. $N_{\Delta}$ is the number of factors $(a \Delta),(b \Delta)$ and $(S \Delta)$ in the decomposition (77). One must have $l \geq k-1$ for $E_{T n, k}, M_{T n, k}$ and $l \geq k$ for $\widetilde{E}_{T n, k}, \widetilde{M}_{T n, k}$, and $i \geq 0$ for all cases. The corresponding graphs contribute to the form factor at order $O\left(q^{d}\right)$ with $d \geq D_{l, i}-N_{\Delta}$ and $D_{l, i}$ from (33).

\begin{tabular}{cccc}
\hline \hline form factor & $k$ & $N_{\Delta}$ & operators \\
\hline$E_{T n, k}$ & odd & $k+1$ & $Q_{n, l, i}^{\lambda \mu}$ \\
$M_{T n, k}$ & even & $k+1$ & $Q_{n, l, i}^{\lambda \mu}$ \\
$\widetilde{E}_{T n, k}$ & even & $k$ & $Q_{n, l, i}^{\lambda \mu}$ \\
$\widetilde{M}_{T n, k}$ & even & $k+2$ & $Q_{n, l, i}^{\lambda \mu}$ \\
\hline \hline
\end{tabular}

To obtain the general form of $\mathcal{O}_{R L, n}^{\lambda \mu}$ and $\mathcal{O}_{L R, n}^{\lambda \mu}$ it is sufficient to construct corresponding operators $\mathcal{O}^{\lambda \mu}$ that involve no $\epsilon$ tensor and either no spin vector or two spin vectors in the form $\left[S_{\lambda}, S_{\mu}\right]$. Operators with one $\epsilon$ tensor and one spin vector can be brought into this form by using the third relation in (30) and rewriting the resulting product of two $\epsilon$ tensors in terms of products of metric tensors. Terms in $\mathcal{O}_{R L, n}^{\lambda \mu}$ and $\mathcal{O}_{L R, n}^{\lambda \mu}$ with an odd total number of $\epsilon$ tensors and spin vectors are then readily obtained by adding the dual operators $\frac{1}{2} i \epsilon^{\lambda \mu}{ }_{\alpha \beta} \mathcal{O}^{\alpha \beta}$ with coefficients determined by the relations (86), using that $\frac{1}{2} i \epsilon^{\lambda \mu}{ }_{\alpha \beta} \frac{1}{2} i \epsilon^{\alpha \beta}{ }_{\gamma \delta} t^{\gamma \delta}=t^{\lambda \mu}$ for any antisymmetric tensor $t^{\lambda \mu}$.

Following the procedure of Section 4.1 we decompose the pion-nucleon part of the operators $Q_{n}^{A, \lambda \mu}(a)$ as

$$
Q_{n, \pi N}^{\lambda \mu}(a)=\sum_{k=0}^{n-1} M^{n-k-1}(a v)^{n-k-1} Q_{n, k}^{\lambda \mu}(a),
$$

where we have omitted superscripts $A$ for ease of writing. The operator $Q_{n, k}^{\lambda \mu}(a)$ is the contraction of a tensor of rank $k+2$ with $k$ vectors $a$ and may not contain any factors $(a v)$. The minimal number of vectors $\partial_{\rho}, \overleftrightarrow{\nabla}_{\rho}, u_{\rho}$ in $Q_{n, k}^{\lambda \mu}(a)$ is $k-1$ and must be accompanied either by the tensor $v^{\lambda}\left[S^{\mu},(a S)\right]-v^{\mu}\left[S^{\lambda},(a S)\right]$ or by its dual $i \epsilon^{\lambda \mu \alpha \beta} v_{\alpha}\left[S_{\beta},(a S)\right]$. In the first case one obtains however the structure $(a v)[(b S),(a S)]$ after contraction with $b_{\lambda} a_{\mu}$, which also appears in $b_{\lambda} a_{\mu}(a v)^{n-k} Q_{n, k-1}^{\lambda \mu}(a)$. An analogous statement holds of course in the case of the dual tensor. We can therefore restrict ourselves to operators $Q_{n, k}^{\lambda \mu}(a)$ with at least $k$ vectors $\partial_{\rho}, \overleftrightarrow{\nabla}_{\rho}, u_{\rho}$, and thus further decompose

$$
Q_{n, k}^{\lambda \mu}(a)=\sum_{i=0}^{\infty} M^{-i} Q_{n, k, i}^{\lambda \mu}(a)
$$

where $Q_{n, k, i}^{\lambda \mu}(a)$ has chiral dimension $k+i$. The power counting for graphs with a certain operator insertion proceeds in close analogy to Section 4.2 and is summarized in Table 2. Comparing the number of factors $(a v)$ in (91) and in the decomposition (77), one obtains the restriction $l \geq k$ for the operators $Q_{n, l, i}^{\lambda \mu}(a)$ that can contribute to $\widetilde{E}_{T n, k}$ and $\widetilde{M}_{T n, k}$. For $E_{T n, k}$ and $M_{T n, k}$ the restriction is $l \geq k-1$, where the case $l=k-1$ requires that the graphs with insertion of $Q_{n, l, i}^{\lambda \mu}(a)$ produce no factors of $v^{\lambda}$ or $v^{\mu}$. 


\subsection{Pure pion operators}

Pionic operators which transform according to (85) can be written as

$$
\mathcal{O}_{R L, n}^{\lambda \mu}(a)=u \mathcal{O} u, \quad \mathcal{O}_{L R, n}^{\lambda \mu}(a)=u^{\dagger} \mathcal{O}^{\prime} u^{\dagger}
$$

where $\mathcal{O}$ and $\mathcal{O}^{\prime}$ are related by a parity transformation, transform like $u_{\mu}$ under chiral rotations, and are constructed from the fields $u_{\mu} \chi_{ \pm}$and covariant derivatives. We can restrict the derivatives to act only on fields inside $\mathcal{O}$ and $\mathcal{O}^{\prime} 7$ With the duality relations (86) one finds that the pure pion part of the operator $Q_{n}^{A, \lambda \mu}(a)$ can be brought into the form

$$
\operatorname{Tr}\left[\tau_{o+}^{A} V^{\lambda \mu}(a)\right]+\frac{i}{2} \epsilon^{\lambda \mu \alpha \beta} \operatorname{Tr}\left[\tau_{o-}^{A} V_{\alpha \beta}(a)\right] \quad \text { or } \quad \operatorname{Tr}\left[\tau_{o-}^{A} A^{\lambda \mu}(a)\right]+\frac{i}{2} \epsilon^{\lambda \mu \alpha \beta} \operatorname{Tr}\left[\tau_{o+}^{A} A_{\alpha \beta}(a)\right],
$$

where $V_{\lambda \mu}$ and $A_{\lambda \mu}$ respectively behave as a tensor or a pseudotensor under parity and are constructed from $u_{\rho}, \nabla_{\rho}$ and $\chi_{ \pm}$without the $\epsilon$ tensor. One readily finds that the terms without $\epsilon$ in (94) couple to an even number and the terms with $\epsilon$ to an odd number of pion fields. $V_{\lambda \mu}(a)$ and $A_{\lambda \mu}(a)$ are tensors of rank $n+1$ contracted with $n-1$ vectors $a$. In the following we consider the terms with the lowest chiral dimension in the pure pion part of $\mathcal{O}_{T n}^{A}(a, b)$. These terms contain no fields $\chi_{ \pm}$and have the vector indices of all $n+1$ factors $u_{\rho}$ or $\nabla_{\rho}$ contracted with either $a$ or $b$.

To calculate matrix elements of these operators between two nucleons or two pions at one-loop accuracy, we only need terms that couple to at most four pions. Terms coupling to three or four pions can appear in tadpole graphs. Such graphs are only nonzero if the pion fields in the operator which couple to the loop have no derivatives acting on them. This is because the corresponding loop integral has a numerator of the form $l_{\rho_{1}} \ldots l_{\rho_{m}}$, where $l$ is the loop momentum. After the loop integration, one obtains zero for odd $m$ and for even $m$ one obtains a combination of metric tensors, which gives zero when the vector indices are contracted with $a$ or $b$.

Since the derivatives with indices $\lambda$ and $\mu$ in the antisymmetric tensor $Q_{n}^{A, \lambda \mu}(a)$ cannot act on the same pion field, one readily finds that operators coupling to one or three pions do not contribute to matrix elements between two nucleons or two pions. For the same reason such operators decouple from matrix elements between the vacuum and a single pion, which reflects the fact that there are no chiral-odd pion distribution amplitudes of twist two.

It remains to construct operators $V_{\lambda \mu}(a)$ and $A_{\lambda \mu}(a)$ from $u_{\rho}$ and $\nabla_{\rho}$, which must have at least one factor $u_{\rho}$ because the covariant derivatives must act on some field to give nonzero, and less than three such factors because of the restriction just discussed. The operators with one factor of $u_{\rho}$ are of the form 8

$$
(a \nabla)^{k_{1}} \nabla^{\lambda}(a \nabla)^{k_{2}} u^{\mu}-(\lambda \leftrightarrow \mu) \quad \text { or } \quad(a \nabla)^{k_{1}} \nabla^{\lambda}(a \nabla)^{k_{2}} \nabla^{\mu}(a \nabla)^{k_{3}}(a u)-(\lambda \leftrightarrow \mu) .
$$

In both cases we can use the commutator identity $\left[\nabla^{\alpha}, \nabla^{\beta}\right] \mathcal{O}=\frac{1}{4}\left(\left[u^{\alpha}, u^{\beta}\right] \mathcal{O}-\mathcal{O}\left[u^{\alpha}, u^{\beta}\right]\right)$ to bring the vectors with indices $\lambda$ and $\mu$ next to each other. The commutator terms do not contribute to the matrix element in question since they involve three or more vectors $u_{\rho}$. The remaining term involves either $\nabla^{\lambda} u^{\mu}-\nabla^{\mu} u^{\lambda}=0$ or $\left[\nabla^{\lambda}, \nabla^{\mu}\right] \ldots(a u)$ and thus do not contribute either.

The only relevant operators contain hence two vectors $u_{\rho}$. According to our above discussion, the $\Gamma_{\rho}$ part of any factor $\nabla_{\rho}$ does not contribute in this case, and the derivative must act on the pion fields in $u_{\rho}$ which already carry a derivative. For the matrix elements in question, $\nabla^{\lambda}(a u)$ is hence equivalent to $(a \nabla) u^{\lambda}$. The same holds of course for the index $\mu$. We thus find that the operators of

\footnotetext{
${ }^{7}$ Other terms can be brought into this form using identities such as $\partial_{\rho}(u \mathcal{O} u)=u\left(\left[\nabla_{\rho}, \mathcal{O}\right]-\frac{i}{2}\left\{u_{\rho}, \mathcal{O}\right\}\right) u$.

${ }^{8}$ For simplicity we write from now on $\nabla_{\mu} \mathcal{O}$ instead of $\left[\nabla_{\mu}, \mathcal{O}\right]$ if $\mathcal{O}$ transforms like $u_{\mu}$ under chiral rotations.
} 
lowest chiral dimension can be written as

$$
Q_{n, \pi}^{A, \lambda \mu}(a)=\frac{F^{2}}{8} \sum_{\substack{k=0 \\ \text { even }}}^{n-1} b_{T n, k} \operatorname{Tr} \tau_{o+}^{A}(i a \nabla)^{k} V_{n, k}^{\lambda \mu}+\ldots
$$

with

$$
V_{n, k}^{\lambda \mu}=u^{\lambda}(2 i a \overleftrightarrow{\nabla})^{n-k-1} u^{\mu}-u^{\mu}(2 i a \overleftrightarrow{\nabla})^{n-k-1} u^{\lambda}
$$

where the ... denote terms not contributing to two-nucleon or two-pion matrix elements at tree level or one loop. We note that the coefficients $b_{T n, k}$ have nonzero mass dimension and are of order $(4 \pi F)^{-1}$ in the sense of chiral power counting. They give the tree-level contribution at order $O\left(q^{0}\right)$ to the pion form factors $B_{T n, k}^{\pi}(t)$ defined in (82),

$$
B_{T n, k}^{\pi(0)}=(-1)^{n+1} 2^{n-k-1} m_{\pi} b_{T n, k},
$$

where $n$ is even in the isosinglet and odd in the isotriplet case. The restriction to even $k$ in (96) corresponds to the one in (82).

We can now apply the power counting formula (37) with $d_{\pi}=n+1$ to the operators just constructed. Taking into account the restrictions of even or odd $n$ or $k$ for the different form factors, we find that the corrections from pion operator insertions start at order $O(q)$ for $\widetilde{M}_{T n, n-1}^{I=1}$ and at order $O\left(q^{2}\right)$ for $M_{T n, n-1}^{I=1}, E_{T n, n-1}^{I=0}$ and $\widetilde{M}_{T n, n-2}^{I=0}$. For all other form factors they start at order $O\left(q^{3}\right)$ or higher.

\section{Results for chiral-odd form factors}

Using the construction described in Section 7, we find pion-nucleon operators

$$
\begin{aligned}
Q_{n, k, 0}^{A, \lambda \mu}= & \widetilde{E}_{T n, k}^{I(0)}(i a \partial)^{k}\left(\bar{N}_{v}\left[S^{\lambda}, S^{\mu}\right] \tau_{o+}^{A} N_{v}+\bar{N}_{v}\left(v^{\lambda} S^{\mu}-v^{\mu} S^{\lambda}\right) \tau_{o-}^{A} N_{v}\right)+\ldots \\
Q_{n, k, 1}^{A, \lambda \mu}= & -\frac{i}{4} M_{T n, k}^{I(0)}(i a \partial)^{k}\left(\left(v^{\lambda} \partial^{\mu}-v^{\mu} \partial^{\lambda}\right) \bar{N}_{v} \tau_{o+}^{A} N_{v}+i \epsilon^{\lambda \mu \alpha \beta} v_{\alpha} \partial_{\beta} \bar{N}_{v} \tau_{o-}^{A} N_{v}\right)+\ldots \\
& +\frac{i}{2} E_{T n, k}^{I(0)}(i a \partial)^{k} \partial_{\rho}\left(v^{\mu} \bar{N}_{v}\left[S^{\lambda}, S^{\rho}\right] \tau_{o+}^{A} N_{v}-v^{\lambda} \bar{N}_{v}\left[S^{\mu}, S^{\rho}\right] \tau_{o+}^{A} N_{v}+\left\{\text { terms with } \tau_{o-}^{A}\right\}\right)+\ldots \\
Q_{n, k, 2}^{A, \lambda \mu}= & -\frac{1}{4} \widetilde{M}_{T n, k}^{I(0)}(i a \partial)^{k} \partial_{\rho}\left(\partial^{\mu} \bar{N}_{v}\left[S^{\lambda}, S^{\rho}\right] \tau_{o+}^{A} N_{v}-\partial^{\lambda} \bar{N}_{v}\left[S^{\mu}, S^{\rho}\right] \tau_{o+}^{A} N_{v}+\left\{\text { terms with } \tau_{o-}^{A}\right\}\right)+\ldots
\end{aligned}
$$

where the ... denote terms with a smaller number of total derivatives. The coefficients in (99) are the tree-level contributions at order $O\left(q^{0}\right)$ to the respective form factors and therefore only nonzero for even or odd $k$ as given in Table 2, The terms with $\tau_{o-}^{A}$ in the last two lines of (99) are rather lengthy and not given here. Indeed, one finds that none of the operators with $\tau_{o-}^{A}$ in (99) contributes in oneloop graphs with pion-nucleon interactions at LO or at NLO. Such graphs have the form of Fig. 11k and give zero for the same reasons discussed after (40) for the case of operators with $\tau_{e-}^{A}$. The discussion at the end of Section 5.1 also applies to the operators with $\tau_{o+}^{A}$ in (99), so that their insertion into graphs with LO pion-nucleon vertices or with one NLO pion-nucleon interaction contributes only to those form factors for which they already provide the tree-level result at order $O\left(q^{0}\right)$.

An operator $Q_{n, l, i}^{\lambda \mu}$ in (99) has at most $l+i$ partial derivatives, so that the condition (36) holds also in the chiral-odd case. Together with the power counting following from Table 2, one again finds 
that one-loop corrections to all form factors start at order $O\left(q^{2}\right)$. One finds that the order $O\left(q^{2}\right)$ corrections to $M_{T n, k}$ and $E_{T n, k}$ come from $Q_{n, k, 1}$, whereas those to $\widetilde{E}_{T n, k}$ and $\widetilde{M}_{T n, k}$ come from $Q_{n, k, 0}$ and $Q_{n, k, 2}$, respectively, with pion-nucleon interactions taken at LO in all cases. Additional order $O\left(q^{2}\right)$ contributions to $\widetilde{M}_{T n, k}$ come from graphs with $Q_{n, k, 0}$ and two pion-nucleon interactions at NLO or one pion-nucleon interaction at NNLO (only the $\tau_{o+}^{A}$ part of the operator is found to contribute). Contributions from the same graphs to $E_{T n, k+1}$ or $M_{T n, k+1}$ are possible by power counting but turn out to be zero. Other contributions at order $O\left(q^{2}\right)$ which are possible by power counting involve at most one pion-nucleon interaction at NLO and do not appear for the reason given at the end of the preceding paragraph: there is no correction to $M_{T n, k}$ or $E_{T n, k}$ from $Q_{n, k+1,0}, Q_{n, k, 0}, Q_{n, k-1,1}$ or $Q_{n, k-1,2}$ and no correction to $\widetilde{M}_{T n, k}$ from $Q_{n, k+1,0}, Q_{n, k+2,0}, Q_{n, k, 1}$ or $Q_{n, k+1,1}$.

Taking into account the graphs with pion operator insertions shown in Fig. 2a and b, we finally find

$$
\begin{aligned}
& M_{T n, k}^{I=0}=M_{T n, k}^{I=0(0)}\left(1-\frac{3 m^{2}}{2(4 \pi F)^{2}} \log \frac{m^{2}}{\mu^{2}}\right)+\ldots \\
& M_{T n, k}^{I=1}=M_{T n, k}^{I=1(0)}\left(1-\frac{m^{2}}{2(4 \pi F)^{2}}\left[\left(6 g_{A}^{2}+1\right) \log \frac{m^{2}}{\mu^{2}}+4 g_{A}^{2}\right]\right)+\delta_{k, n-1} M_{T n, n-1}^{I=1(2, \pi)}(t)+\ldots, \\
& E_{T n, k}^{I=0}=E_{T n, k}^{I=0(0)}\left(1-\frac{3 m^{2}}{2(4 \pi F)^{2}}\left(2 g_{A}^{2}+1\right) \log \frac{m^{2}}{\mu^{2}}\right)+\delta_{k, n-1} E_{T n, n-1}^{I=0(2, \pi)}(t)+\ldots, \\
& E_{T n, k}^{I=1}=E_{T n, k}^{I=1(0)}\left(1-\frac{m^{2}}{2(4 \pi F)^{2}}\left[\left(4 g_{A}^{2}+1\right) \log \frac{m^{2}}{\mu^{2}}+4 g_{A}^{2}\right]\right)+\ldots \\
& \widetilde{E}_{T n, k}^{I=0}=\widetilde{E}_{T n, k}^{I=0(0)}\left(1-\frac{3 m^{2}}{2(4 \pi F)^{2}}\left(2 g_{A}^{2}+1\right) \log \frac{m^{2}}{\mu^{2}}\right)+\ldots, \\
& \widetilde{E}_{T n, k}^{I=1}=\widetilde{E}_{T n, k}^{I=1(0)}\left(1-\frac{m^{2}}{2(4 \pi F)^{2}}\left[\left(4 g_{A}^{2}+1\right) \log \frac{m^{2}}{\mu^{2}}+4 g_{A}^{2}\right]\right)+\ldots
\end{aligned}
$$

and

$$
\begin{aligned}
\widetilde{M}_{T n, k}^{I=0}= & \widetilde{M}_{T n, k}^{I=0(0)}\left(1-\frac{3 m^{2}}{2(4 \pi F)^{2}}\left(2 g_{A}^{2}+1\right) \log \frac{m^{2}}{\mu^{2}}\right)+\widetilde{E}_{T n, k}^{I=0(0)} \frac{m^{2} g_{A}^{2}}{(4 \pi F)^{2}} \log \frac{m^{2}}{\mu^{2}} \\
& +\delta_{k, n-2} \widetilde{M}_{T n, n-2}^{I=0(2, \pi)}(t)+\ldots, \\
\widetilde{M}_{T n, k}^{I=1}= & \widetilde{M}_{T n, k}^{I=1(0)}\left(1-\frac{m^{2}}{2(4 \pi F)^{2}}\left[\left(4 g_{A}^{2}+1\right) \log \frac{m^{2}}{\mu^{2}}+4 g_{A}^{2}\right]\right)-\widetilde{E}_{n, k}^{I=1(0)} \frac{m^{2} g_{A}^{2}}{3(4 \pi F)^{2}} \log \frac{m^{2}}{\mu^{2}} \\
& +\delta_{k, n-1}\left[\widetilde{M}_{T n, n-1}^{I=1(1, \pi)}(t)+\widetilde{M}_{T n, n-1}^{I=1(2, \pi)}(t)\right]+\ldots,
\end{aligned}
$$

where for brevity we have written ... to denote analytic terms proportional to $m^{2}$ or $t$ and corrections of order $O\left(q^{3}\right)$. The analytic terms are due to higher-order tree-level insertions as specified below (34). The contributions from pion operator insertions read

$$
\begin{aligned}
E_{T n, n-1}^{I=0(2, \pi)}(t) & =-\frac{1}{4} \widetilde{M}_{T n, n-2}^{I=0(2, \pi)}(t) \\
\widetilde{M}_{T n, n-2}^{I=0(2, \pi)}(t) & =-\frac{3 g_{A}^{2}}{(4 \pi F)^{2}} \sum_{\substack{j=2 \\
\text { even }}}^{n}(j-1) M b_{T n, n-j} \int_{-1}^{1} d \eta \eta^{j-2} m^{2}(\eta) \log \frac{m^{2}(\eta)}{\mu^{2}}
\end{aligned}
$$


with $n$ even in the isosinglet case and

$$
\begin{aligned}
& M_{T n, n-1}^{I=1(2, \pi)}(t)=\frac{1}{4(4 \pi F)^{2}} \sum_{\substack{j=1 \\
\text { odd }}}^{n} M b_{T n, n-j}\left\{4 g_{A}^{2} m^{2} \log \frac{m^{2}}{\mu^{2}}\right. \\
& \left.\quad+\int_{-1}^{1} d \eta \eta^{j-1}\left[g_{A}^{2}\left(2 m^{2}-t\right)\left(\log \frac{m^{2}(\eta)}{\mu^{2}}+1\right)-\left(g_{A}^{2}-1\right) m^{2}(\eta) \log \frac{m^{2}(\eta)}{\mu^{2}}\right]\right\} \\
& \widetilde{M}_{T n, n-1}^{I=1(1, \pi)}(t)=-\frac{\pi M g_{A}^{2}}{(4 \pi F)^{2}} \sum_{j=1}^{n} M b_{T n, n-j} \int_{-1}^{1} d \eta \eta^{j-1} m(\eta), \\
& \widetilde{M}_{T n, n-1}^{I=1(2, \pi)}(t)=-\frac{1}{4(4 \pi F)^{2}} \sum_{j=1}^{n} M b_{T n, n-j}\left\{4 g_{A}^{2} m^{2} \log \frac{m^{2}}{\mu^{2}}\right. \\
& \left.\quad+\int_{-1}^{1} d \eta \eta^{j-1}\left[g_{A}^{2}\left(2 m^{2}-t\right)\left(\log \frac{m^{2}(\eta)}{\mu^{2}}+1\right)+\left(g_{A}^{2}-1-4 M c_{4}\right) m^{2}(\eta) \log \frac{m^{2}(\eta)}{\mu^{2}}\right]\right\}
\end{aligned}
$$

with $n$ odd in the isotriplet sector. As remarked in [14, the corrections from pion operator insertions are very similar for the chiral-odd and chiral-even form factors. We find a correspondence

$$
\widetilde{M}_{T n, n-2}^{I=0} \leftrightarrow-M_{n, n-2}^{I=0}, \quad \widetilde{M}_{T n, n-1}^{I=1} \leftrightarrow \frac{1}{2} M_{n, n-1}^{I=1}, \quad M_{T n, n-1}^{I=1} \leftrightarrow \frac{1}{2} E_{n, n-1}^{I=1}
$$

for the terms in (102) and (103) when interchanging $M b_{T n, n-j} \leftrightarrow 2^{-j} j A_{n, n-j}^{\pi(0)}$.

Let us compare our results (100) to (103) to those in Ref. 14, which gives the corrections of order $O(q)$ for $\widetilde{M}_{T n, k}^{I=1}$ and of order $O\left(q^{2}\right)$ for all other form factors 9 We agree with the expressions given there, except for the corrections from nucleon operator insertions without a factor $g_{A}^{2}$ in the isosinglet form factors, which are absent in [14, and for the corresponding term in $M_{T n, k}^{I=1}$, where we have a different coefficient. These corrections are due to the tadpole graph in Fig. 1 b, with the pion-nucleon vertex generated by the two-pion terms in the expansion (89) of $\tau_{o+}^{0}$ and $\tau_{o+}^{a}$. Since this vertex has no spin or momentum structure, the corresponding corrections must be the same for all form factors with a given isospin.

Let us finally give the corrections of order $O\left(q^{2}\right)$ to the chiral-odd GPDs of the pion. They are given by the one-loop graphs shown in Figure 3 with insertion of the pion operators (96) and from tree-level insertions of operators with chiral dimension $n+3$. For the form factors (82) we find

$$
B_{T n, k}^{\pi}(t)=B_{T n, k}^{\pi(0)}\left(1-\frac{3 m^{2}}{2(2 \pi F)^{2}} \log \frac{m^{2}}{\mu^{2}}\right)+\ldots
$$

for even $n$, and

$$
\begin{aligned}
B_{T n, k}^{\pi}(t)= & B_{T n, k}^{\pi(0)}\left(1-\frac{m^{2}}{2(4 \pi F)^{2}} \log \frac{m^{2}}{\mu^{2}}\right) \\
& +\delta_{k, n-1} \frac{1}{(4 \pi F)^{2}} \sum_{\substack{j=1 \\
\text { odd }}}^{n} 2^{-j} B_{T n, n-j}^{\pi(0)} \int_{-1}^{1} d \eta \eta^{j-1} m^{2}(\eta) \log \frac{m^{2}(\eta)}{\mu^{2}}+\ldots
\end{aligned}
$$

\footnotetext{
${ }^{9}$ The tensor form factors in 14 are related to those introduced here by $M^{T} \simeq \widetilde{E}_{T}, E^{T} \simeq M_{T} / 2, W^{T} \simeq-4 \widetilde{M}_{T}($ all up to terms suppressed by factors of order $\left.\Delta^{2} / M^{2}\right)$ and by $C^{T}=E_{T}$.
} 


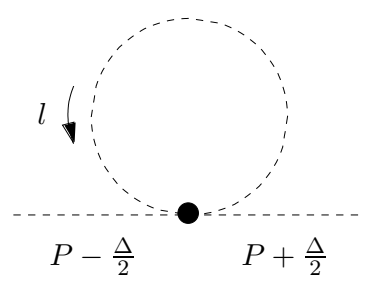

a

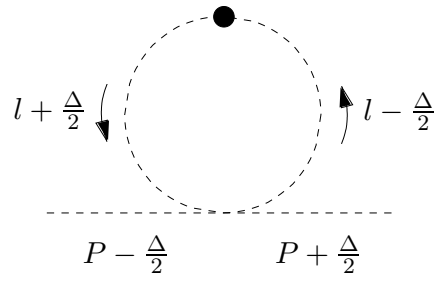

$\mathrm{b}$

Figure 3: One-loop graphs contributing to two-pion matrix elements of the pion operator $Q_{n, \pi}^{A, \lambda \mu}(a)$ in (96). The operator insertion is denoted by a black blob.

for odd $n$, where the ... stand for analytic terms from tree-level graphs and for corrections of order $O\left(q^{4}\right)$. The corrections going with $\log m^{2} / \mu^{2}$ are due to the tadpole graph in Fig. [3a and are independent of $k$. The term involving an integral over $\eta$ is due to the graph of Fig. 3b and can only occur for $k=n-1$ (and thus only in the isotriplet case). This is because the operator insertion on the pion line cannot produce any factor $(a P)$ and the four-pion vertex can only produce one such factor after the loop integration.

\section{Results for moments of nucleon and pion GPDs}

In this section, we rewrite our results in terms of the form factors $A_{n, k}, B_{n, k}, C_{n}, \widetilde{A}_{n, k}, \widetilde{B}_{n, k}$ and $A_{T n, k}$, $\bar{B}_{T n, k}, \widetilde{A}_{T n, k}, \widetilde{B}_{T n, k}$, which describe the moments of GPDs in commonly used parameterizations. We give expressions for the value and the first derivative of each form factor at $t=0$, which should be useful for applications in lattice QCD. The corrections obtained from graphs with pion-nucleon operator insertions are completely specified in this way, because they are independent of $t$. In the following we will use the abbreviation $\Lambda_{\chi}=4 \pi F$.

For a convenient overview of results we also reproduce the expressions for isosinglet distributions from [13] here. Together with (9), (10) and the expressions in Section 5, we find that up to corrections of order $O\left(\mathrm{~m}^{3}\right)$ the chiral-even vector form factors at $t=0$ have the form

$$
\begin{aligned}
A_{n, k}^{I=0}(0)= & A_{n, k}^{I=0(0)}+A_{n, k}^{I=0(2, m)} m^{2}, \\
B_{n, k}^{I=0}(0)= & B_{n, k}^{I=0(0)}-\left(A_{n, k}^{I=0(0)}+B_{n, k}^{I=0(0)}\right) \frac{3 m^{2} g_{A}^{2}}{\Lambda_{\chi}^{2}} \log \frac{m^{2}}{\mu^{2}}+B_{n, k}^{I=0(2, m)} m^{2}+\delta_{k, n-2} B_{n, n-2}^{I=0(2, \pi)}(0), \\
C_{n}^{I=0}(0)= & C_{n}^{I=0(0)}+C_{n}^{I=0(2, m)} m^{2}+C_{n}^{I=0(1, \pi)}(0)+C_{n}^{I=0(2, \pi)}(0), \\
A_{n, k}^{I=1}(0)= & A_{n, k}^{I=1(0)}\left\{1-\frac{m^{2}}{\Lambda_{\chi}^{2}}\left[\left(3 g_{A}^{2}+1\right) \log \frac{m^{2}}{\mu^{2}}+2 g_{A}^{2}\right]\right\}+A_{n, k}^{I=1(2, m)} m^{2}+\delta_{k, n-1} A_{n, n-1}^{I=1(2, \pi)}(0), \\
B_{n, k}^{I=1}(0)= & B_{n, k}^{I=1(0)}\left\{1-\frac{m^{2}}{\Lambda_{\chi}^{2}}\left[\left(3 g_{A}^{2}+1\right) \log \frac{m^{2}}{\mu^{2}}+2 g_{A}^{2}\right]\right\}+\left(A_{n, k}^{I=1(0)}+B_{n, k}^{I=1(0)}\right) \frac{m^{2} g_{A}^{2}}{\Lambda_{\chi}^{2}} \log \frac{m^{2}}{\mu^{2}} \\
& +B_{n, k}^{I=1(2, m)} m^{2}+\delta_{k, n-1}\left[B_{n, n-1}^{I=1(1, \pi)}(0)+B_{n, n-1}^{I=1(2, \pi)}(0)\right], \\
C_{n}^{I=1}(0)= & C_{n}^{I=1(0)}\left\{1-\frac{m^{2}}{\Lambda_{\chi}^{2}}\left[\left(3 g_{A}^{2}+1\right) \log \frac{m^{2}}{\mu^{2}}+2 g_{A}^{2}\right]\right\}+C_{n}^{I=1(2, m)} m^{2} .
\end{aligned}
$$


The labeling of coefficients with superscripts $(0),(2, m),(1, \pi)$ and $(2, \pi)$ follows the same pattern as in Sections 5 and 8 . The contributions from pion operator insertions read

$$
\begin{aligned}
& B_{n, n-2}^{I=0(2, \pi)}(0)=\frac{6 m^{2} g_{A}^{2}}{\Lambda_{\chi}^{2}} \log \frac{m^{2}}{\mu^{2}} \sum_{\substack{j=2 \\
\text { even }}}^{n} 2^{-j} j A_{n, n-j}^{\pi(0)}, \\
& C_{n}^{I=0(1, \pi)}(0)=\frac{3 \pi m M g_{A}^{2}}{2 \Lambda_{\chi}^{2}} \sum_{\substack{j=2 \\
\text { even }}}^{n} 2^{-j} \frac{j}{j+1} A_{n, n-j}^{\pi(0)}, \\
& C_{n}^{I=0(2, \pi)}(0)=-\frac{3 m^{2} g_{A}^{2}}{2 \Lambda_{\chi}^{2}} \log \frac{m^{2}}{\mu^{2}} \sum_{\substack{j=2 \\
\text { even }}}^{n} 2^{-j} j A_{n, n-j}^{\pi(0)}+\frac{12 m^{2}}{\Lambda_{\chi}^{2}}\left[\frac{g_{A}^{2}}{8}-M c_{1}\left(\log \frac{m^{2}}{\mu^{2}}+1\right)\right. \\
&\left.\quad+\frac{3}{4} M c_{2} \log \frac{m^{2}}{\mu^{2}}+M c_{3}\left(\log \frac{m^{2}}{\mu^{2}}+\frac{1}{2}\right)\right] \sum_{\substack{j=2 \\
\text { even }}}^{n} 2^{-j} \frac{j}{j+1} A_{n, n-j}^{\pi(0)},
\end{aligned}
$$

where $n$ is even, and

$$
\begin{aligned}
A_{n, n-1}^{I=1(2, \pi)}(0)= & \frac{2 m^{2} g_{A}^{2}}{\Lambda_{\chi}^{2}} \log \frac{m^{2}}{\mu^{2}} \sum_{\substack{j=1 \\
\text { odd }}}^{n} 2^{-j} j A_{n, n-j}^{\pi(0)}+\frac{m^{2}}{\Lambda_{\chi}^{2}}\left[\left(g_{A}^{2}+1\right) \log \frac{m^{2}}{\mu^{2}}+2 g_{A}^{2}\right] \sum_{\substack{j=1 \\
\text { odd }}}^{n} 2^{-j} A_{n, n-j}^{\pi(0)}, \\
B_{n, n-1}^{I=1(1, \pi)}(0)= & -\frac{4 \pi m M g_{A}^{2}}{\Lambda_{\chi}^{2}} \sum_{\substack{j=1 \\
\text { odd }}}^{n} 2^{-j} A_{n, n-j}^{\pi(0)}, \\
B_{n, n-1}^{I=1(2, \pi)}(0)= & -\frac{4 m^{2} g_{A}^{2}}{\Lambda_{\chi}^{2}} \log \frac{m^{2}}{\mu^{2}} \sum_{j=1}^{n} 2^{-j} j A_{n, n-j}^{\pi(0)} \\
& -\frac{4 m^{2}}{\Lambda_{\chi}^{2}}\left[g_{A}^{2}\left(\log \frac{m^{2}}{\mu^{2}}+1\right)-M c_{4} \log \frac{m^{2}}{\mu^{2}}\right] \sum_{j=1}^{n} 2^{-j} A_{n, n-j}^{\pi(0)},
\end{aligned}
$$

where $n$ is odd. The $A_{n, n-j}^{\pi(0)}$ are the leading terms in the chiral expansion of the pion form factors in (48) and fulfill the relations [12

$$
\sum_{\substack{j=2 \\ \text { even }}}^{n} 2^{-j} A_{n, n-j}^{\pi(0)}=-A_{n, n}^{\pi(0)} \quad \text { for even } n, \quad \sum_{\substack{j=1 \\ \text { odd }}}^{n} 2^{-j} A_{n, n-j}^{\pi(0)}=B_{n}^{\pi(0)} \quad \text { for odd } n,
$$

where $B_{n}^{\pi(0)}$ is the $n$-th moment of the pion distribution amplitude to leading order in the chiral expansion, as introduced in Section 5.3. Estimates for the values of the low-energy constants $c_{i}$ appearing in (108) and (109) can be found in [30].

For the axial form factors we have

$$
\begin{aligned}
& \widetilde{A}_{n, k}^{I=0}(0)=\widetilde{A}_{n, k}^{I=0(0)}\left\{1-\frac{3 m^{2} g_{A}^{2}}{\Lambda_{\chi}^{2}}\left[\log \frac{m^{2}}{\mu^{2}}+1\right]\right\}+\widetilde{A}_{n, k}^{I=0(2, m)} m^{2}, \\
& \widetilde{B}_{n, k}^{I=0}(0)=\widetilde{B}_{n, k}^{I=0(0)}\left\{1-\frac{3 m^{2} g_{A}^{2}}{\Lambda_{\chi}^{2}}\left[\log \frac{m^{2}}{\mu^{2}}+1\right]\right\}-\widetilde{A}_{n, k}^{I=0(0)} \frac{m^{2} g_{A}^{2}}{\Lambda_{\chi}^{2}} \log \frac{m^{2}}{\mu^{2}}+\widetilde{B}_{n, k}^{I=0(2, m)} m^{2},
\end{aligned}
$$




$$
\begin{array}{rlrl}
\widetilde{A}_{n, k}^{I=1}(0)= & \widetilde{A}_{n, k}^{I=1(0)}\left\{1-\frac{m^{2}}{\Lambda_{\chi}^{2}}\left[\left(2 g_{A}^{2}+1\right) \log \frac{m^{2}}{\mu^{2}}+g_{A}^{2}\right]\right\}+\widetilde{A}_{n, k}^{I=1(2, m)} m^{2}, \\
\widetilde{B}_{n, k}^{I=1}(0)= & \widetilde{B}_{n, k}^{I=1(0)}\left\{1-\frac{m^{2}}{\Lambda_{\chi}^{2}}\left[\left(2 g_{A}^{2}+1\right) \log \frac{m^{2}}{\mu^{2}}+g_{A}^{2}\right]\right\} & \\
& +\widetilde{A}_{n, k}^{I=1(0)} \frac{m^{2} g_{A}^{2}}{3 \Lambda_{\chi}^{2}} \log \frac{m^{2}}{\mu^{2}}+\widetilde{B}_{n, k}^{I=1(2, m)} m^{2} & \text { for } k<n-1
\end{array}
$$

with corrections of order $O\left(m^{3}\right)$, and

$$
\widetilde{B}_{n, n-1}^{I=1}(0)=B_{n}^{\pi} \frac{4 M^{2} g_{A}}{m_{\pi}^{2}}\left(1-2 m_{\pi}^{2} g_{A}^{-1} d_{18}\right)+\widetilde{B}_{n, n-1}^{I=1(0)}+O(m) \quad \text { for odd } n .
$$

Note that the implicit pion mass dependence from $B_{n}^{\pi}, M, g_{A}$ and $m_{\pi}$ is relevant within the accuracy of this expression. Numerical estimates of the low-energy constant $d_{18}$ are given in [31]. The derivative of $\widetilde{B}_{n, n-1}^{I=1}(t)$ at $t=0$ reads

$$
\partial_{t} \widetilde{B}_{n, n-1}^{I=1}(0)=B_{n}^{\pi} \frac{4 M^{2} g_{A}}{m_{\pi}^{4}}\left(1-2 m_{\pi}^{2} g_{A}^{-1} d_{18}\right)+O\left(m^{-1}\right)
$$

where the order $O\left(m^{-1}\right)$ corrections are due to terms of the form $O\left(q^{3}\right) /\left(m_{\pi}^{2}-t\right)$ in $\widetilde{B}_{n, n-1}^{I=1}(t)$. Using $B_{1}^{\pi}=1$, we obtain a ratio

$$
\frac{\partial_{t} \widetilde{B}_{n, n-1}^{I=1}(0)}{\partial_{t} \widetilde{B}_{1,0}^{I=1}(0)}=B_{n}^{\pi}+O\left(m^{3}\right)
$$

which involves only physical matrix elements and is independent of any low-energy constants. It would be interesting to test this relation in lattice QCD calculations, as this would indicate how well the chiral expansion works at a given pion mass.

The derivatives at $t=0$ of the remaining chiral-even form factors have nonanalytic contributions in the pion mass only for

$$
\begin{aligned}
\partial_{t} B_{n, n-2}^{I=0}(0) & =B_{n, n-2}^{I=0(2, t)}+\partial_{t} B_{n, n-2}^{I=0(2, \pi)}(0), \\
\partial_{t} C_{n}^{I=0}(0) & =C_{n}^{I=0(2, t)}+\partial_{t} C_{n}^{I=0(1, \pi)}(0)+\partial_{t} C_{n}^{I=0(2, \pi)}(0) \\
\partial_{t} A_{n, n-1}^{I=1}(0) & =A_{n, n-1}^{I=1(2, t)}+\partial_{t} A_{n, n-1}^{I=1(2, \pi)}(0), \\
\partial_{t} B_{n, n-1}^{I=1}(0) & =B_{n, n-1}^{I=1(2, t)}+\partial_{t} B_{n, n-1}^{I=1(1, \pi)}(0)+\partial_{t} B_{n, n-1}^{I=1(2, \pi)}(0),
\end{aligned}
$$

with corrections of order $O(m)$, where

$$
\begin{aligned}
& \partial_{t} B_{n, n-2}^{I=0(2, \pi)}(0)=-\frac{3 g_{A}^{2}}{\Lambda_{\chi}^{2}}\left(\log \frac{m^{2}}{\mu^{2}}+1\right) \sum_{\substack{j=2 \\
\text { even }}}^{n} 2^{-j} \frac{j}{j+1} A_{n, n-j}^{\pi(0)}, \\
& \partial_{t} C_{n}^{I=0(1, \pi)}(0)=-\frac{M}{m} \frac{\pi g_{A}^{2}}{8 \Lambda_{\chi}^{2}} \sum_{\substack{j=2 \\
\text { even }}}^{n} 2^{-j} \frac{j(5 j+14)}{(j+1)(j+3)} A_{n, n-j}^{\pi(0)},
\end{aligned}
$$




$$
\begin{aligned}
\partial_{t} C_{n}^{I=0(2, \pi)}(0) & =-\frac{3 g_{A}^{2}}{4 \Lambda_{\chi}^{2}}\left(\log \frac{m^{2}}{\mu^{2}}+3\right) \sum_{\substack{j=2 \\
\text { even }}}^{n} 2^{-j} \frac{j}{j+1} A_{n, n-j}^{\pi(0)}+\frac{2}{\Lambda_{\chi}^{2}}\left[\frac{g_{A}^{2}}{8}+M c_{1}\right. \\
& \left.-\frac{3}{4} M c_{2}\left(\log \frac{m^{2}}{\mu^{2}}+1\right)-M c_{3}\left(\log \frac{m^{2}}{\mu^{2}}+\frac{3}{2}\right)\right] \sum_{\substack{j=2 \\
\text { even }}}^{n} 2^{-j} \frac{j(j+4)}{(j+1)(j+3)} A_{n, n-j}^{\pi(0)}
\end{aligned}
$$

with $n$ even, and

$$
\begin{aligned}
\partial_{t} A_{n, n-1}^{I=1(2, \pi)}(0)= & -\frac{g_{A}^{2}}{\Lambda_{\chi}^{2}}\left(\log \frac{m^{2}}{\mu^{2}}+1\right) \sum_{\substack{j=1 \\
\text { odd }}}^{n} 2^{-j} A_{n, n-j}^{\pi(0)} \\
& +\frac{1}{2 \Lambda_{\chi}^{2}}\left[\left(g_{A}^{2}-1\right) \log \frac{m^{2}}{\mu^{2}}-\left(g_{A}^{2}+1\right)\right] \sum_{\substack{j=1 \\
\text { odd }}}^{n} 2^{-j} \frac{1}{j+2} A_{n, n-j}^{\pi(0)}, \\
\partial_{t} B_{n, n-1}^{I=1(1, \pi)}(0)= & \frac{M}{m} \frac{\pi g_{A}^{2}}{\Lambda_{\chi}^{2}} \sum_{j=1}^{n} 2^{-j} \frac{1}{j+2} A_{n, n-j}^{\pi(0)}, \\
\partial_{t} B_{n, n-1}^{I=1(2, \pi)}(0)= & \frac{2 g_{A}^{2}}{\Lambda_{\chi}^{2}}\left(\log \frac{m^{2}}{\mu^{2}}+1\right) \sum_{j=1}^{n} 2^{-j} A_{n, n-j}^{\pi(0)} \underset{\text { odd }}{ } \\
& +\frac{2}{\Lambda_{\chi}^{2}}\left[g_{A}^{2}-M c_{4}\left(\log \frac{m^{2}}{\mu^{2}}+1\right)\right] \sum_{j=1}^{n} 2^{-j} \frac{1}{j+2} A_{n, n-j}^{\pi(0)} .
\end{aligned}
$$

with $n$ odd. All other chiral-even nucleon form factors receive only corrections from pion-nucleon operators, so that their derivatives at $t=0$ are given by the appropriate coefficients with superscript $(2, t)$, which are due to tree-level contributions.

For the chiral-odd nucleon form factors at $t=0$ we find

$$
\begin{aligned}
A_{T n, k}^{I=0}(0)= & A_{T n, k}^{I=0(0)}\left\{1-\frac{3 m^{2}}{2 \Lambda_{\chi}^{2}}\left(2 g_{A}^{2}+1\right) \log \frac{m^{2}}{\mu^{2}}\right\}+A_{T n, k}^{I=0(2, m)} m^{2}, \\
\bar{B}_{T n, k}^{I=0}(0)= & \bar{B}_{T n, k}^{I=0(0)}\left\{1-\frac{3 m^{2}}{2 \Lambda_{\chi}^{2}}\left(2 g_{A}^{2}+1\right) \log \frac{m^{2}}{\mu^{2}}\right\} \\
& +\left(A_{T n, k}^{I=0(0)}+\bar{B}_{T n, k}^{I=0(0)}\right) \frac{3 m^{2} g_{A}^{2}}{\Lambda_{\chi}^{2}} \log \frac{m^{2}}{\mu^{2}}+\bar{B}_{T n, k}^{I=0(2, m)} m^{2}, \\
\widetilde{A}_{T n, k}^{I=0}(0)= & \widetilde{A}_{T n, k}^{I=0(0)}\left\{1-\frac{3 m^{2}}{2 \Lambda_{\chi}^{2}}\left(2 g_{A}^{2}+1\right) \log \frac{m^{2}}{\mu^{2}}\right\}+\left(A_{T n, k}^{I=0(0)}+\frac{3}{2} \bar{B}_{T n, k}^{I=0(0)}\right) \frac{m^{2} g_{A}^{2}}{\Lambda_{\chi}^{2}} \log \frac{m^{2}}{\mu^{2}} \\
& +\widetilde{A}_{T n, k}^{I=0(2, m)} m^{2}+\delta_{k, n-2} \widetilde{A}_{T n, n-2}^{I=0(2, \pi)}(0), \\
\widetilde{B}_{T n, k}^{I=0}(0)= & \widetilde{B}_{T n, k}^{I=0(0)}\left\{1-\frac{3 m^{2}}{2 \Lambda_{\chi}^{2}}\left(2 g_{A}^{2}+1\right) \log \frac{m^{2}}{\mu^{2}}\right\}+\widetilde{B}_{T n, k}^{I=0(2, m)} m^{2}+\delta_{k, n-1} \widetilde{B}_{T n, n-1}^{I=0(2, \pi)}(0),
\end{aligned}
$$




$$
\begin{aligned}
A_{T n, k}^{I=1}(0)= & A_{T n, k}^{I=1(0)}\left\{1-\frac{m^{2}}{2 \Lambda_{\chi}^{2}}\left[\left(4 g_{A}^{2}+1\right) \log \frac{m^{2}}{\mu^{2}}+4 g_{A}^{2}\right]\right\}+A_{T n, k}^{I=1(2, m)} m^{2}, \\
\bar{B}_{T n, k}^{I=1}(0)= & \bar{B}_{T n, k}^{I=1(0)}\left\{1-\frac{m^{2}}{2 \Lambda_{\chi}^{2}}\left[\left(4 g_{A}^{2}+1\right) \log \frac{m^{2}}{\mu^{2}}+4 g_{A}^{2}\right]\right\} \\
& -\left(A_{T n, k}^{I=1(0)}+\bar{B}_{T n, k}^{I=1(0)}\right) \frac{m^{2} g_{A}^{2}}{\Lambda_{\chi}^{2}} \log \frac{m^{2}}{\mu^{2}}+\bar{B}_{T n, k}^{I=1(2, m)} m^{2}+\delta_{k, n-1} \bar{B}_{T n, n-1}^{I=1(2, \pi)}(0), \\
\widetilde{A}_{T n, k}^{I=1}(0)= & \widetilde{A}_{T n, k}^{I=1(0)}\left\{1-\frac{m^{2}}{2 \Lambda_{\chi}^{2}}\left[\left(4 g_{A}^{2}+1\right) \log \frac{m^{2}}{\mu^{2}}+4 g_{A}^{2}\right]\right\}-\left(A_{T n, k}^{I=1(0)}+\frac{3}{2} \bar{B}_{T n, k}^{I=1(0)}\right) \frac{m^{2} g_{A}^{2}}{3 \Lambda_{\chi}^{2}} \log \frac{m^{2}}{\mu^{2}} \\
& +\widetilde{A}_{T n, k}^{I=1(2, m)} m^{2}+\delta_{k, n-1}\left[\widetilde{A}_{T n, n-1}^{I=1(1, \pi)}(0)+\widetilde{A}_{T n, n-1}^{I=1(2, \pi)}(0)\right], \\
\widetilde{B}_{T n, k}^{I=1}(0)= & \widetilde{B}_{T n, k}^{I=1(0)}\left\{1-\frac{m^{2}}{2 \Lambda_{\chi}^{2}}\left[\left(4 g_{A}^{2}+1\right) \log \frac{m^{2}}{\mu^{2}}+4 g_{A}^{2}\right]\right\}+\widetilde{B}_{T n, k}^{I=1(2, m)} m^{2}
\end{aligned}
$$

with corrections of order $O\left(\mathrm{~m}^{3}\right)$, where

$$
\begin{aligned}
& \widetilde{A}_{T n, n-2}^{I=0(2, \pi)}(0)=\frac{3 m^{2} g_{A}^{2}}{\Lambda_{\chi}^{2}} \log \frac{m^{2}}{\mu^{2}} \sum_{\substack{j=2 \\
\text { even }}}^{n} M b_{T n, n-j}, \\
& \widetilde{B}_{T n, n-1}^{I=0(2, \pi)}(0)=\frac{1}{2} \widetilde{A}_{T n, n-2}^{I=0(2, \pi)}(0)
\end{aligned}
$$

with $n$ even, and

$$
\begin{aligned}
\bar{B}_{T n, n-1}^{I=1(2, \pi)}(0)= & \frac{m^{2} g_{A}^{2}}{\Lambda_{\chi}^{2}} \log \frac{m^{2}}{\mu^{2}} \sum_{\substack{\text { odd } \\
\text { odd }}}^{n} M b_{T n, n-j}+\frac{m^{2}}{2 \Lambda_{\chi}^{2}}\left[\left(g_{A}^{2}+1\right) \log \frac{m^{2}}{\mu^{2}}+2 g_{A}^{2}\right] \sum_{\substack{j=1 \\
\text { odd }}}^{n} \frac{1}{j} M b_{T n, n-j}, \\
\widetilde{A}_{T n, n-1}^{I=1(1, \pi)}(0)= & \frac{\pi m M g_{A}^{2}}{\Lambda_{\chi}^{2}} \sum_{j=1}^{n} \frac{1}{j} M b_{T n, n-j}, \\
\widetilde{A}_{T n, n-1}^{I=1(2, \pi)}(0)= & \frac{m^{2} g_{A}^{2}}{\Lambda_{\chi}^{2}} \log \frac{m^{2}}{\mu^{2}} \sum_{j=1}^{n} M b_{T n, n-j} \\
& +\frac{m^{2}}{\Lambda_{\chi}^{2}}\left[g_{A}^{2}\left(\log \frac{m^{2}}{\mu^{2}}+1\right)-M c_{4} \log \frac{m^{2}}{\mu^{2}}\right] \sum_{j=1}^{n} \frac{1}{j} M b_{T n, n-j}
\end{aligned}
$$

with $n$ odd. Our results for $A_{n, 0}^{I=1}(0), \widetilde{A}_{n, 0}^{I=1}(0)$ and $A_{T n, 0}^{I=1}(0)$ reproduce the expressions in [32] for the distributions of unpolarized, longitudinally and transversely polarized quarks and antiquarks in the nucleon. The derivatives at $t=0$ of the following form factors have nonanalytic contributions in the pion mass:

$$
\begin{aligned}
& \partial_{t} \widetilde{A}_{T n, n-2}^{I=0}(0)=\widetilde{A}_{T n, n-2}^{I=0(2, t)}+\partial_{t} \widetilde{A}_{T n, n-2}^{I=0(2, \pi)}(0) \\
& \partial_{t} \widetilde{B}_{T n, n-1}^{I=0}(0)=\widetilde{B}_{T n, n-1}^{I=0(2, t)}+\partial_{t} \widetilde{B}_{T n, n-1}^{I=0(2, \pi)}(0) \\
& \partial_{t} \bar{B}_{T n, n-1}^{I=1}(0)=\bar{B}_{T n, n-1}^{I=1(2, t)}+\partial_{t} \bar{B}_{T n, n-1}^{I=1(2, \pi)}(0) \\
& \partial_{t} \widetilde{A}_{T n, n-1}^{I=1}(0)=\widetilde{A}_{T n, n-1}^{I=1(2, t)}+\partial_{t} \widetilde{A}_{T n, n-1}^{I=1(1, \pi)}(0)+\partial_{t} \widetilde{A}_{T n, n-1}^{I=1(2, \pi)}(0),
\end{aligned}
$$


where corrections are of order $O(m)$ and

$$
\begin{aligned}
& \partial_{t} \widetilde{A}_{T n, n-2}^{I=0(2, \pi)}(0)=-\frac{3 g_{A}^{2}}{2 \Lambda_{\chi}^{2}}\left(\log \frac{m^{2}}{\mu^{2}}+1\right) \sum_{\substack{j=2 \\
\text { even }}}^{n} \frac{1}{j+1} M b_{T n, n-j}, \\
& \partial_{t} \widetilde{B}_{T n, n-1}^{I=0(2, \pi)}(0)=\frac{1}{2} \partial_{t} \widetilde{A}_{T n, n-2}^{I=0(2, \pi)}(0)
\end{aligned}
$$

with even $n$ and

$$
\begin{aligned}
\partial_{t} \bar{B}_{T n, n-1}^{I=1(2, \pi)}(0)= & -\frac{g_{A}^{2}}{2 \Lambda_{\chi}^{2}}\left(\log \frac{m^{2}}{\mu^{2}}+1\right) \sum_{\substack{j=1 \\
\text { odd }}}^{n} \frac{1}{j} M b_{T n, n-j} \\
& +\frac{1}{4 \Lambda_{\chi}^{2}}\left[\left(g_{A}^{2}-1\right) \log \frac{m^{2}}{\mu^{2}}-\left(g_{A}^{2}+1\right)\right] \sum_{\substack{j=1 \\
\text { odd }}}^{n} \frac{1}{j(j+2)} M b_{T n, n-j}, \\
\partial_{t} \widetilde{A}_{T n, n-1}^{I=1(1, \pi)}(0)= & -\frac{M}{m} \frac{\pi g_{A}^{2}}{4 \Lambda_{\chi}^{2}} \sum_{j=1}^{n} \frac{1}{j(j+2)} M b_{T n, n-j}, \\
\partial_{t} \widetilde{A}_{T n, n-1}^{I=1(2, \pi)}(0)= & -\frac{g_{A}^{2}}{2 \Lambda_{\chi}^{2}}\left(\log \frac{m^{2}}{\mu^{2}}+1\right) \sum_{\substack{j=1 \\
\text { odd }}}^{n} \frac{1}{j} M b_{T n, n-j} \\
& -\frac{1}{2 \Lambda_{\chi}^{2}}\left[g_{A}^{2}-M c_{4}\left(\log \frac{m^{2}}{\mu^{2}}+1\right)\right] \sum_{\substack{j=1 \\
\text { odd }}}^{n} \frac{1}{j(j+2)} M b_{T n, n-j}
\end{aligned}
$$

with odd $n$. As a consequence of the relations (104), we find the following correspondence between the corrections (119), (120), (122), (123) from pion loop insertions to chiral-odd form factors and their chiral-even counterparts (108), (109), (116), (117):

$$
\widetilde{A}_{T n, n-2}^{I=0} \leftrightarrow \frac{1}{2} B_{n, n-2}^{I=0} \quad \widetilde{A}_{T n, n-1}^{I=1} \leftrightarrow-\frac{1}{4} B_{n, n-1}^{I=1} \quad \bar{B}_{T n, n-1}^{I=1} \leftrightarrow \frac{1}{2} A_{n, n-1}^{I=1}
$$

when the low-energy constants are interchanged as $M b_{T n, n-j} \leftrightarrow 2^{-j} j A_{n, n-j}^{\pi(0)}$.

Let us also give the expressions of form factors and their derivatives at $t=0$ for the moments of pion GPDs. For the chiral-even moments, the expressions given in [12] result in

$$
A_{n, k}^{\pi}(0)= \begin{cases}A_{n, k}^{\pi(0)}+A_{n, k}^{\pi(2, m)} m^{2}+\delta_{k, n} A_{n, n}^{\pi(l, 2)} & \text { for even } n \\ A_{n, k}^{\pi(0)}\left(1-\frac{m^{2}}{\Lambda_{\chi}^{2}} \log \frac{m^{2}}{\mu^{2}}\right)+A_{n, k}^{\pi(2, m)} m^{2}+\delta_{k, n-1} A_{n, n-1}^{\pi(l, 2)} & \text { for odd } n\end{cases}
$$

with corrections of order $O\left(m^{4}\right)$ and

$$
\begin{aligned}
& A_{n, n}^{\pi(l, 2)}=-\frac{m^{2}}{2 \Lambda_{\chi}^{2}}\left(\log \frac{m^{2}}{\mu^{2}}+1\right) \sum_{\substack{j=2 \\
\text { even }}}^{n} 2^{-j} \frac{j}{j+1} A_{n, n-j}^{\pi(0)}, \\
& A_{n, n-1}^{\pi(l, 2)}=\frac{2 m^{2}}{\Lambda_{\chi}^{2}} \log \frac{m^{2}}{\mu^{2}} \sum_{\substack{j=1 \\
\text { odd }}}^{n} 2^{-j} A_{n, n-j}^{\pi(0)} .
\end{aligned}
$$


Using the relation (110) one thus has

$$
A_{n, n-1}^{\pi}(0)=A_{n, n-1}^{\pi(0)}+\frac{m^{2}}{\Lambda_{\chi}^{2}} \log \frac{m^{2}}{\mu^{2}}\left[2 B_{n}^{\pi(0)}-A_{n, n-1}^{\pi(0)}\right]+A_{n, n-1}^{\pi(2, m)} m^{2}+O\left(m^{4}\right)
$$

with $n$ odd. For the chiral-odd moments we have with (105) and (106)

$$
B_{T n, k}^{\pi}(0)= \begin{cases}B_{T n, k}^{\pi(0)}\left(1-\frac{3 m^{2}}{2 \Lambda_{\chi}^{2}} \log \frac{m^{2}}{\mu^{2}}\right)+B_{T n, k}^{\pi(2, m)} m^{2} & \text { for even } n, \\ B_{T n, k}^{\pi(0)}\left(1-\frac{m^{2}}{2 \Lambda_{\chi}^{2}} \log \frac{m^{2}}{\mu^{2}}\right)+B_{T n, k}^{\pi(2, m)} m^{2}+\delta_{k, n-1} B_{T n, n-1}^{\pi(l, 2)} & \text { for odd } n\end{cases}
$$

where corrections are again of order $O\left(m^{4}\right)$ and

$$
B_{T n, n-1}^{\pi(l, 2)}=\frac{2 m^{2}}{\Lambda_{\chi}^{2}} \log \frac{m^{2}}{\mu^{2}} \sum_{\substack{j=1 \\ \text { odd }}}^{n} 2^{-j} \frac{1}{j} B_{T n, n-j}^{\pi(0)} .
$$

The only nonanalytic contributions in the pion mass for the derivatives of form factors are

$$
\begin{aligned}
\partial_{t} A_{n, n}^{\pi}(0) & =A_{n, n}^{\pi(2, t)}+\frac{1}{\Lambda_{\chi}^{2}}\left(\log \frac{m^{2}}{\mu^{2}}+1\right) \sum_{\substack{j=2 \\
\text { even }}}^{n} 2^{-j} \frac{j}{j+1} A_{n, n-j}^{\pi(0)}+\frac{1}{12 \Lambda_{\chi}^{2}} \sum_{\substack{j=2 \\
\text { even }}}^{n} 2^{-j} \frac{j(j+4)}{(j+1)(j+3)} A_{n, n-j}^{\pi(0)} \\
\partial_{t} A_{n, n-1}^{\pi}(0) & =A_{n, n-1}^{\pi(2, t)}-\frac{1}{\Lambda_{\chi}^{2}}\left(\log \frac{m^{2}}{\mu^{2}}+1\right) \sum_{\substack{j=1 \\
\text { odd }}}^{n} 2^{-j} \frac{1}{j+2} A_{n, n-j}^{\pi(0)} \\
\partial_{t} B_{T n, n-1}^{\pi}(0) & =B_{T n, n-1}^{\pi(2, t)}-\frac{1}{\Lambda_{\chi}^{2}}\left(\log \frac{m^{2}}{\mu^{2}}+1\right) \sum_{\substack{j=1 \\
\text { odd }}}^{n} 2^{-j} \frac{1}{j(j+2)} B_{T n, n-j}^{\pi(0)}
\end{aligned}
$$

where the second index is always even and corrections are of order $O\left(\mathrm{~m}^{2}\right)$.

Let us finally take a look at moments of parton distributions whose values are fixed by quantum numbers for arbitrary values of the pion mass, see e.g. [32, 33. For $A_{1,0}^{\pi}(0)$ one readily finds that the explicit chiral logarithm in (125) cancels against the one in (126). This is required to ensure the quark number sum rule

$$
A_{1,0}^{\pi}(0)=\int_{0}^{1} d x\left(u_{\pi}-\bar{u}_{\pi}-d_{\pi}+\bar{d}_{\pi}\right)=2
$$

in the pion, which also implies $A_{1,0}^{\pi(0)}=2$ and $A_{1,0}^{\pi(2, m)}=0$. With this one also finds that our result (107) is consistent with the quark number sum rules

$$
A_{1,0}^{I=0}(0)=\int_{0}^{1} d x(u-\bar{u}+d-\bar{d})=3, \quad A_{1,0}^{I=1}(0)=\int_{0}^{1} d x(u-\bar{u}-d+\bar{d})=1,
$$

in the proton, provided that $A_{1,0}^{I=0(0)}=3, A_{1,0}^{I=1(0)}=1$ and $A_{1,0}^{I=0(2, m)}=A_{1,0}^{I=1(2, m)}=0$.

\section{Summary}

In this paper and its companion [13] we have calculated the chiral corrections to the full set of twisttwo generalized parton distributions in the nucleon, using heavy-baryon chiral perturbation theory. 
For each form factor parameterizing the moments of these distributions, our results include the order $O\left(q^{2}\right)$ relative to its lowest-order expression. We have presented a detailed account of the power counting and of the operators that can contribute to the chiral order we consider. We find that the operator structure is relatively simple in the basis of form factors specified by (9) and (78). With the exception of $\widetilde{M}_{n, k}$ and $\widetilde{M}_{T n, k}$ only those pion-nucleon operator insertions contribute to the loop corrections of a given form factor which already provide its lowest-order expression at tree-level. Furthermore, only operators with $\tau_{e+}^{A}$ or $\tau_{o+}^{A}$ from (28) and (88) contribute, but not those with $\tau_{e-}^{A}$ or $\tau_{o-}^{A}$. Our analysis also shows that these simplifications will no longer hold at higher orders in the chiral expansion.

Expressing our results in the basis of form factors parameterizing the moments of the usual nucleon GPDs, we find that with the exception of $A_{n, k}^{I=0}$ and $C_{n}^{I=0}$ all form factors receive chiral corrections from loop graphs with nucleon operator insertions (see Fig. 1). They are of relative order $O\left(q^{2}\right)$ and contain logarithmic terms $m^{2} \log \left(m^{2} / \mu^{2}\right)$, but are independent of $t$ and of the indices $n, k$. In several cases these corrections involve a mixing between different form factors: $B_{n, k}$ receives corrections involving not only its own lowest-order expression but also the one of $A_{n, k}$, as seen in (107). Likewise, there are corrections to $\widetilde{B}_{n, k}$ from $\widetilde{A}_{n, k}$, to $\bar{B}_{T n, k}$ from $A_{T n, k}$, and to $\widetilde{A}_{T n, k}$ from $A_{T n, k}+\frac{3}{2} \bar{B}_{T n, k}$. We note that no such mixing occurs for the linear combinations $A_{n, k}+B_{n, k}$ and $A_{T n, k}+\bar{B}_{T n, k}$.

Further corrections are due to loop graphs with pion operator insertions (see Fig. 2 a and b). They only occur for form factors which are accompanied by the maximal number of vectors $\Delta_{\mu}$ in the decomposition of the associated matrix element, or by one factor less. Due to the quantum number restrictions for pion operators, they only occur for even $n$ in the isosinglet and for odd $n$ in the isotriplet sector. Corrections starting at order $O(q)$ are obtained for $C_{n}^{I=0}, B_{n, n-1}^{I=1}$ and $\widetilde{A}_{T n, n-1}^{I=1}$, and corrections starting at order $O\left(q^{2}\right)$ for $B_{n, n-2}^{I=0}, \widetilde{A}_{T n, n-2}^{I=0}, \widetilde{B}_{T n, n-1}^{I=0}, A_{n, n-1}^{I=1}$ and $\bar{B}_{T n, n-1}^{I=1}$. To order $O\left(q^{2}\right)$, the corrections for $C_{n}^{I=0}$ involve the low-energy constants $c_{1}, c_{2}, c_{3}$ from the pion-nucleon Lagrangian (18), whereas those for $B_{n, n-1}^{I=1}$ and $\widetilde{A}_{T n, n-1}^{I=1}$ involve $c_{4}$. The corrections from pion operator insertions depend on $t$. They are responsible for a nonanalytic pion mass dependence of the derivatives of

form factors at $t=0$, namely a $1 / m$ behavior for $\partial_{t} C_{n}^{I=0}(0), \partial_{t} B_{n, n-1}^{I=1}(0)$ and $\partial_{t} \widetilde{A}_{T n, n-1}^{I=1}(0)$ and a $\log \left(m^{2} / \mu^{2}\right)$ behavior in the other cases. We note that these corrections also determine the onset of the two-pion cut at timelike $t$ for the form factors in question.

The pseudoscalar form factors $\widetilde{B}_{n, n-1}^{I=1}$ receive corrections from one-pion exchange (see Fig. 22k). They take the very simple form (68) when expressed in terms of physical quantities. In particular, we find that the ratio $\partial_{t} \widetilde{B}_{n, n-1}^{I=1}(0) / \partial_{t} \widetilde{B}_{1,0}^{I=1}(0)$ of derivatives is given by the moment $B_{n}^{\pi}$ of the pion distribution amplitude, with corrections of order $\mathrm{m}^{3}$. It would be interesting to test this prediction of chiral symmetry in lattice QCD calculations.

We have finally evaluated the corrections to the chiral-odd pion GPDs at order $O\left(q^{2}\right)$, thus complementing the calculation [12] for the chiral-even sector. A compilation of our results for the values and derivatives at $t=0$ of all moments of nucleon and pion GPDs is given in Section 9 .

\section{Acknowledgments}

We are grateful to U.-G. Meißner for clarifying discussions. This work is supported by the Helmholtz Association, contract number VH-NG-004. 


\section{References}

[1] D. Müller, D. Robaschik, B. Geyer, F. M. Dittes and J. Hořejši, Fortschr. Phys. 42 (1994) 101 hep-ph/9812448.

[2] X. D. Ji, Phys. Rev. Lett. 78 (1997) 610 hep-ph/9603249.

[3] A. V. Radyushkin, Phys. Rev. D56 (1997) 5524 [hep-ph/9704207].

[4] X. D. Ji, J. Phys. G 24 (1998) 1181 hep-ph/9807358.

[5] K. Goeke, M. V. Polyakov and M. Vanderhaeghen, Prog. Part. Nucl. Phys. 47 (2001) 401 hep-ph/0106012.

[6] M. Diehl, Phys. Rept. 388 (2003) 41 hep-ph/0307382.

[7] A. V. Belitsky and A. V. Radyushkin, Phys. Rept. 418 (2005) 1 hep-ph/0504030].

[8] M. Diehl and Ph. Hägler, Eur. Phys. J. C 44 (2005) 87 hep-ph/0504175;

M. Diehl et al. [QCDSF Collaboration], hep-ph/0511032.

[9] M. Burkardt, Phys. Rev. D 72 (2005) 094020 hep-ph/0505189].

[10] K. Kumerički, D. Müller, K. Passek-Kumerički and A. Schäfer, hep-ph/0605237;

D. Müller, Phys. Lett. B 634 (2006) 227 [hep-ph/0510109].

[11] M. Göckeler et al., [QCDSF Collaboration], Phys. Rev. D 71 (2005) 034508 hep-lat/0303019];

J. D. Ashley, D. B. Leinweber, A. W. Thomas and R. D. Young, Eur. Phys. J. A 19 (2004) 9 hep-lat/0308024];

C. Alexandrou, G. Koutsou, J. W. Negele and A. Tsapalis, Phys. Rev. D 74 (2006) 034508 hep-lat/0605017.

[12] M. Diehl, A. Manashov and A. Schäfer, Phys. Lett. B 622 (2005) 69 hep-ph/0505269].

[13] M. Diehl, A. Manashov and A. Schäfer, Eur. Phys. J. A 29 (2006) 315 hep-ph/0608113.

[14] S. I. Ando, J. W. Chen and C. W. Kao, hep-ph/0602200 v4.

[15] R. G. Edwards et al. [LHPC Collaboration], hep-lat/0610007.

[16] M. Göckeler et al. [QCDSF-UKQCD Collaboration], PoS LAT2005 (2006) 055;

Nucl. Phys. Proc. Suppl. 153 (2006) 146 hep-lat/0512011.

[17] J. Gasser, M. E. Sainio and A. Švarc, Nucl. Phys. B 307 (1988) 779.

[18] V. Bernard, H. W. Fearing, T. R. Hemmert and U.-G. Meißner, Nucl. Phys. A 635 (1998) 121, Erratum ibid. A 642 (1998) 563 hep-ph/9801297].

[19] V. Bernard, N. Kaiser and U.-G. Meißner, Int. J. Mod. Phys. E 4 (1995) 193 hep-ph/9501384.

[20] S. Steininger, U.-G. Meißner and N. Fettes, JHEP 9809 (1998) 008 hep-ph/9808280;

J. Kambor and M. Mojžiš, JHEP 9904 (1999) 031 hep-ph/9901235.

[21] N. Fettes, U.-G. Meißner and S. Steininger, Nucl. Phys. A 640 (1998) 199 hep-ph/9803266.

[22] J. Gasser and H. Leutwyler, Annals Phys. 158 (1984) 142. 
[23] G. Ecker and M. Mojžiš, Phys. Lett. B 365 (1996) 312 [hep-ph/9508204].

[24] V. Bernard and U.-G. Meißner, Phys. Lett. B 639 (2006) 278 hep-lat/0605010.

[25] L. Mankiewicz, G. Piller and A. Radyushkin, Eur. Phys. J. C10 (1999) 307 hep-ph/9812467.

[26] M. Penttinen, M. V. Polyakov and K. Goeke, Phys. Rev. D62 (2000) 014024 hep-ph/9909489.

[27] V. Bernard, L. Elouadrhiri and U.-G. Meißner, J. Phys. G 28 (2002) R1 hep-ph/0107088].

[28] M. Diehl, Eur. Phys. J. C 19 (2001) 485 hep-ph/0101335.

[29] Ph. Hägler, Phys. Lett. B 594 (2004) 164 hep-ph/0404138.

[30] U.-G. Meißner, PoS LAT2005 (2006) 009 hep-lat/0509029.

[31] N. Fettes and U.-G. Meißner, Nucl. Phys. A 676 (2000) 311 hep-ph/0002162];

E. Epelbaum, U.-G. Meißner and W. Glöckle, Nucl. Phys. A 714 (2003) 535 nucl-th/0207089].

[32] J. W. Chen and X. D. Ji, Phys. Lett. B 523 (2001) 107 hep-ph/0105197.

[33] D. Arndt and M. J. Savage, Nucl. Phys. A 697 (2002) 429 nucl-th/0105045]. 